\title{
STABILIZED FINITE ELEMENT METHODS BASED ON MULTISCALE ENRICHMENT FOR THE STOKES PROBLEM*
}

\author{
RODOLFO ARAYA ${ }^{\dagger}$, GABRIEL R. BARRENECHEA ${ }^{\dagger}$, AND FRÉDÉRIC VALENTIN ${ }^{\ddagger}$
}

\begin{abstract}
This work concerns the development of stabilized finite element methods for the Stokes problem considering nonstable different (or equal) order of velocity and pressure interpolations. The approach is based on the enrichment of the standard polynomial space for the velocity component with multiscale functions which no longer vanish on the element boundary. On the other hand, since the test function space is enriched with bubble-like functions, a Petrov-Galerkin approach is employed. We use such a strategy to propose stable variational formulations for continuous piecewise linear in velocity and pressure and for piecewise linear/piecewise constant interpolation pairs. Optimal order convergence results are derived and numerical tests validate the proposed methods.
\end{abstract}

Key words. Stokes equation, multiscale functions, SIMPLEST element, bubble function

AMS subject classification. $65 \mathrm{~N} 30$

DOI. $10.1137 / 050623176$

1. Introduction. Finite element solution of the Stokes problem poses the basic problem of satisfying the discrete Babuska-Brezzi (or inf-sup) condition (see [24] and the references therein). This is indeed a restriction from the point of view of implementation since equal order velocity and pressure spaces do not satisfy this condition. On the other hand, the minimal space to imagine, namely continuous piecewise linear polynomials for the velocity and piecewise constant polynomials for the pressure, does not satisfy this condition either.

Several solutions have been proposed to overcome this restriction, starting with that in [11] and the first consistent method in [28]. Moreover, in [23, 27, 29, 34] the possibility of considering discontinuous spaces for the pressure was considered and justified. On the other hand, in [14, 13], the idea from [16] has been used to propose a new kind of stabilized finite element methods, with stabilizing terms now containing only jump terms across the interelement boundaries. For an overview of stabilized finite element methods for the Stokes problem, see [19] and [5].

On the other hand, the theoretical justification of stabilized methods has become a subject of interest in the last decade. In $[2,3,4,31]$, the connection between stabilized finite element methods and Galerkin methods enriched with bubble functions has been used to propose new stabilized finite element methods for Stokes-like and linearized Navier-Stokes problems. Also, in [22] macro bubbles were used to derive a method analogous to the locally stabilized method from [29] containing jump terms across the interelement boundaries of the macroelements. In the resulting method, the stabilizing

${ }^{*}$ Received by the editors January 24, 2005; accepted for publication (in revised form) August 2, 2005; published electronically March 7, 2006. A part of this work was done during the stay of the third author at the Departamento de Ingeniería Matemática of Universidad de Concepción and the stay of the first and second authors at LNCC, Petrópolis, Brazil, in the framework of the joint Chile (CONICYT)-Brazil (CNPq) project 2003-4-173 (Chile)-690221/02-9 (Brazil).

http://www.siam.org/journals/sinum/44-1/62317.html

†Departamento de Ingeniería Matemática, Universidad de Concepción, Casilla 160-C, Concepción, Chile (raraya@ing-mat.udec.cl, gbarrene@ing-mat.udec.cl). The first author is partially supported by FONDECYT project 1040595. The second author is partially supported by CONICYT-Chile through FONDECYT project 1030674 and FONDAP Program on Applied Mathematics.

‡Departamento de Matemática Aplicada, Laboratório Nacional de Computação Científica, Av. Getúlio Vargas, 333, 25651-070 Petrópolis - RJ, Brazil (valentin@lncc.br). 
terms are defined over the macroelements, and there is no error analysis or numerical validation of the method. All these works used the so-called bubble condensation procedure, i.e., eliminating the bubble function at the element level and writing the method as the Galerkin part, plus a term derived from the influence of the bubble functions on the formulation. A particular kind of bubble enrichment of the velocity space is the so-called residual-free bubble (RFB) method (cf. $[7,8,12])$, in which the bubble function is now the solution of a problem containing the residual of the continuous equation at the element level (see $[9,10,32]$ for the a priori error analysis). This bubble part may be analytically condensed or numerically computed. In the latter case this procedure leads to the two-level finite element method.

The imposition of a zero boundary condition on the element boundary for the RFB has led to some numerical problems. Solutions for these problems have been proposed by relaxing the zero boundary condition, such as the discontinuous enrichment method [18] (for the Helmholtz equation), and, more recently, the multiscale finite element method; see [21], where the main idea may be found, and [20], where the a priori error analysis is performed (for the reaction-diffusion equation, an a posteriori error estimator based on this idea has been proposed and analyzed in [1]). A particularity of such methods is that a Petrov-Galerkin strategy is proposed, in which the test function space is enriched with bubble functions in order to have a local problem containing the residual of the momentum equation on the right-hand side. A special boundary condition (related to the one used in $[25,26,15]$ ) is imposed in order to solve these local problems analytically. The resulting method is of Petrov-Galerkin type, in which the trial function space is generated by a basis formed by the addition of usual polynomial basis functions and enrichment functions from the solution of the differential problem in each element (which are now, unlike the RFB, known analytically, and hence the method is not of a two-level finite element method type), and in which the test function space is the standard polynomial space.

The purpose of this work is to use the multiscale approach from $[21,20]$, combined with the static condensation procedure, in order to propose new stabilized finite element methods for the Stokes problem. We proceed as in [21], defining an enrichment function for the trial space for the velocity that no longer vanishes on the element boundary (and hence it is not a bubble function), and then we split it into a bubble part and a function being a harmonic extension of the boundary condition. This boundary condition comes from the solution of an elliptic ODE containing a part of the differential operator at the boundary, and a jump term as the right-hand side. Depending on the jump term chosen, this procedure will lead to different methods. Both functions are condensed, and hence we obtain a method which includes the usual Galerkin-Least-Squares (GLS) stabilizing terms at the element level, plus a positive jump term on the interelement boundaries, each one with a proper stabilization parameter. One special feature of these new methods is that the previously mentioned ODE at the element boundary may be solved analytically, and hence the stabilization parameter associated with the jump terms is known exactly.

The plan of the paper is as follows. In section 2 we present the general framework and derive a general form of the method. In sections 3 and 4 this framework is applied to derive concrete stabilized finite element methods for two families of interpolation spaces, namely $\mathbb{P}^{1} / \mathbb{P}^{0}$ and continuous $\mathbb{P}^{1} / \mathbb{P}^{1}$ elements. For both cases optimal order a priori error estimates are derived for the natural norms of the unknowns, plus some extra control on the norm of the jumps appearing in the formulation. As we already mentioned, if we change the right-hand side on the boundary condition, we can derive a new method. This is done in section 5 , where we give an alternative enrichment 
strategy leading to another family of methods, whose analysis is analogous to that of sections 3 and 4, and which contains a boundary term containing the residual of the Cauchy stress tensor on the internal edges of the triangulation. Numerical experiments confirming the theoretical results and comparing the performance of all the methods are presented in section 6 , and some final remarks and conclusions are given in section 7 .

2. The model problem and the general framework. Let $\Omega$ be an open bounded domain in $\mathbb{R}^{2}$ with polygonal boundary, $f \in L^{2}(\Omega)^{2}$ and consider the following Stokes problem:

$$
\begin{aligned}
-\nu \Delta \boldsymbol{u}+\nabla p & =\boldsymbol{f}, \quad \nabla \cdot \boldsymbol{u}=0 \quad \text { in } \Omega, \\
\boldsymbol{u} & =\mathbf{0} \quad \text { on } \partial \Omega,
\end{aligned}
$$

where $\nu \in \mathbb{R}^{+}$is the fluid viscosity.

Now let $\left\{\mathcal{T}_{h}\right\}_{h>0}$ be a family of regular triangulations of $\Omega$, built up using triangles $K$ with boundary $\partial K$. Let also $\mathcal{E}_{h}$ be the set of internal edges of the triangulation, $h_{K}:=\operatorname{diam}(K)$ and $h:=\max \left\{h_{K}: K \in \mathcal{T}_{h}\right\}$. Let $V_{h}$ be the usual finite element space of continuous piecewise polynomials of degree $k, 1 \leq k \leq 2$ with zero trace on $\partial \Omega$. Let also $Q_{h}$ be a space of piecewise polynomials of degree $l, 0 \leq l \leq 1$, which may be continuous or discontinuous in $\Omega$ and which belong to $L_{0}^{2}(\Omega)$. Let $H^{m}\left(\mathcal{T}_{h}\right)$ and $H_{0}^{m}\left(\mathcal{T}_{h}\right)(m \geq 1)$ be the spaces of functions whose restriction to $K \in \mathcal{T}_{h}$ belongs to $H^{m}(K)$ and $H_{0}^{m}(K)$, respectively. Furthermore, $(\cdot, \cdot)_{D}$ stands for the inner product in $L^{2}(D)$ (or in $L^{2}(D)^{2}$ or $L^{2}(D)^{2 \times 2}$, when necessary), and we denote by $\|\cdot\|_{s, D}\left(|\cdot|_{s, D}\right)$ the norm (seminorm) in $H^{s}(D)$ (or $H^{s}(D)^{2}$, if necessary). As usual, $H^{0}(D)=L^{2}(D)$, and $|\cdot|_{0, D}=\|\cdot\|_{0, D}$.

In order to propose a Petrov-Galerkin method for the Stokes problem (1), let $E_{h} \subset H_{0}^{1}(\Omega)$ be a finite-dimensional space, called a multiscale space, such that $V_{h} \cap$ $E_{h}=\{0\}$. Then, we propose the following Petrov-Galerkin scheme for (1): Find $\boldsymbol{u}_{1}+\boldsymbol{u}_{e} \in\left[V_{h} \oplus E_{h}\right]^{2}$ and $p \in Q_{h}$ such that

$$
\nu\left(\nabla\left(\boldsymbol{u}_{1}+\boldsymbol{u}_{e}\right), \nabla \boldsymbol{v}_{h}\right)_{\Omega}-\left(p, \nabla \cdot \boldsymbol{v}_{h}\right)_{\Omega}+\left(q, \nabla \cdot\left(\boldsymbol{u}_{1}+\boldsymbol{u}_{e}\right)\right)_{\Omega}=\left(\boldsymbol{f}, \boldsymbol{v}_{h}\right)_{\Omega}
$$

for all $\boldsymbol{v}_{h} \in\left[V_{h} \oplus H_{0}^{1}\left(\mathcal{T}_{h}\right)\right]^{2}$ and all $q \in Q_{h}$. Now, this Petrov-Galerkin scheme is equivalent to the following system:

$$
\begin{gathered}
\nu\left(\nabla\left(\boldsymbol{u}_{1}+\boldsymbol{u}_{e}\right), \nabla \boldsymbol{v}_{1}\right)_{\Omega}-\left(p, \nabla \cdot \boldsymbol{v}_{1}\right)_{\Omega}+\left(q, \nabla \cdot\left(\boldsymbol{u}_{1}+\boldsymbol{u}_{e}\right)\right)_{\Omega} \\
=\left(\boldsymbol{f}, \boldsymbol{v}_{1}\right)_{\Omega} \quad \forall\left(\boldsymbol{v}_{1}, q\right) \in V_{h}^{2} \times Q_{h}, \\
\nu\left(\nabla\left(\boldsymbol{u}_{1}+\boldsymbol{u}_{e}\right), \nabla \boldsymbol{v}_{b}\right)_{K}-\left(p, \nabla \cdot \boldsymbol{v}_{b}\right)_{K}=\left(\boldsymbol{f}, \boldsymbol{v}_{b}\right)_{K} \quad \forall \boldsymbol{v}_{b} \in H_{0}^{1}(K)^{2} \forall K \in \mathcal{T}_{h} .
\end{gathered}
$$

Equation (3) above is equivalent to

$$
\left(-\nu \Delta \boldsymbol{u}_{e}, \boldsymbol{v}_{b}\right)_{K}=\left(\boldsymbol{f}+\nu \Delta \boldsymbol{u}_{1}-\nabla p, \boldsymbol{v}_{b}\right)_{K} \quad \forall \boldsymbol{v}_{b} \in H_{0}^{1}(K)^{2},
$$

which, in strong form, may be written as

$$
-\nu \Delta \boldsymbol{u}_{e}=\boldsymbol{f}+\nu \Delta \boldsymbol{u}_{1}-\nabla p \text { in } K .
$$

Now, this differential problem must be completed with boundary conditions. For reasons that will become clear in what follows, we will impose the following boundary 
condition on $\boldsymbol{u}_{e}$ :

$$
\boldsymbol{u}_{e}=\boldsymbol{g}_{e} \quad \text { on each } Z \subset \partial K,
$$

where $\boldsymbol{g}_{e}=\mathbf{0}$ if $Z \subset \partial \Omega$, and $\boldsymbol{g}_{e}$ is the solution of

$$
\begin{aligned}
-\nu \partial_{s s} \boldsymbol{g}_{e} & =\frac{1}{h_{Z}} \llbracket \nu \partial_{\boldsymbol{n}} \boldsymbol{u}_{1}+p \mathbf{I} \cdot \boldsymbol{n} \rrbracket \quad \text { in } Z, \\
\boldsymbol{g}_{e} & =\mathbf{0} \text { at the nodes, }
\end{aligned}
$$

on the internal edges, where $h_{Z}=|Z|, \boldsymbol{n}$ is the normal outward vector on $\partial K, \partial_{\boldsymbol{s}}$, and $\partial_{\boldsymbol{n}}$ are the tangential and normal derivative operators, respectively, $\llbracket v \rrbracket$ stands for the jump of $v$ across $Z$, and $\mathbf{I}$ is the $\mathbb{R}^{2 \times 2}$ identity matrix.

REMARK 2.1. Both the shape of the jump term and the $h_{Z}^{-1}$ coefficient on the boundary condition have been suggested by the error analysis. On the other hand, if we impose as the right-hand side in (6) the residual of the Cauchy stress tensor on $\partial K$, we have another class of methods. This alternative will be analyzed in section 5 .

Now, on each $K \in \mathcal{T}_{h}$, we can write $\left.\boldsymbol{u}_{e}\right|_{K}=\boldsymbol{u}_{e}^{K}+\boldsymbol{u}_{e}^{\partial K}$, where

$$
\begin{aligned}
-\nu \Delta \boldsymbol{u}_{e}^{K} & =\boldsymbol{f}+\nu \Delta \boldsymbol{u}_{1}-\nabla p \quad \text { in } K, \\
\boldsymbol{u}_{e}^{K} & =\mathbf{0} \quad \text { on } \partial K,
\end{aligned}
$$

and

$$
\begin{aligned}
-\nu \Delta \boldsymbol{u}_{e}^{\partial K} & =\mathbf{0} \quad \text { in } K, \\
\boldsymbol{u}_{e}^{\partial K} & =\boldsymbol{g}_{e} \quad \text { on } \partial K,
\end{aligned}
$$

where $\boldsymbol{g}_{e}$ is the solution of (6). Such differential problems are well posed, and (3) is immediately satisfied.

In this way, we can define two operators $\mathcal{M}_{K}: L^{2}(K)^{2} \rightarrow H_{0}^{1}(K)^{2}$ and $\mathcal{B}_{K}$ : $L^{2}(\partial K)^{2} \rightarrow H^{1}(K)^{2}$ such that

$$
\boldsymbol{u}_{e}^{K}=\frac{1}{\nu} \mathcal{M}_{K}\left(\boldsymbol{f}+\nu \Delta \boldsymbol{u}_{1}-\nabla p\right) \quad \forall K \in \mathcal{T}_{h}
$$

and

$$
\boldsymbol{u}_{e}^{\partial K}=\frac{1}{\nu} \mathcal{B}_{K}\left(\llbracket \nu \partial_{\boldsymbol{n}} \boldsymbol{u}_{1}+p \mathbf{I} \cdot \boldsymbol{n} \rrbracket\right) \quad \forall K \in \mathcal{T}_{h} .
$$

Next, since the enriched part $\boldsymbol{u}_{e}$ is fully identified through (9)-(10) (or, equivalently, by (7)-(8)), we can perform statical condensation to derive a stabilized finite element method for our problem (1). First, integrating by parts, we have, on each $K \in \mathcal{T}_{h}$,

$$
\begin{gathered}
\nu\left(\nabla \boldsymbol{u}_{e}, \nabla \boldsymbol{v}_{1}\right)_{K}=-\nu\left(\boldsymbol{u}_{e}, \Delta \boldsymbol{v}_{1}\right)_{K}+\left(\boldsymbol{u}_{e}, \nu \partial_{\boldsymbol{n}} \boldsymbol{v}_{1}\right)_{\partial K}, \\
\left(q, \nabla \cdot \boldsymbol{u}_{e}\right)_{K}=-\left(\boldsymbol{u}_{e}, \nabla q\right)_{K}+\left(\boldsymbol{u}_{e}, q \mathbf{I} \cdot \boldsymbol{n}\right)_{\partial K} .
\end{gathered}
$$

Using these identities we can rewrite (2) in the following way:

$$
\begin{gathered}
\nu\left(\nabla \boldsymbol{u}_{1}, \nabla \boldsymbol{v}_{1}\right)_{\Omega}+\sum_{K \in \mathcal{T}_{h}}\left[-\left(\boldsymbol{u}_{e}, \nu \Delta \boldsymbol{v}_{1}\right)_{K}+\left(\boldsymbol{u}_{e}, \nu \partial_{\boldsymbol{n}} \boldsymbol{v}_{1}\right)_{\partial K}\right]-\left(p, \nabla \cdot \boldsymbol{v}_{1}\right)_{\Omega} \\
+\left(q, \nabla \cdot \boldsymbol{u}_{1}\right)_{\Omega}+\sum_{K \in \mathcal{T}_{h}}\left[-\left(\boldsymbol{u}_{e}, \nabla q\right)_{K}+\left(\boldsymbol{u}_{e}, q \mathbf{I} \cdot \boldsymbol{n}\right)_{\partial K}\right]=\left(\boldsymbol{f}, \boldsymbol{v}_{1}\right)_{\Omega},
\end{gathered}
$$


which implies

$$
\begin{gathered}
\nu\left(\nabla \boldsymbol{u}_{1}, \nabla \boldsymbol{v}_{1}\right)_{\Omega}-\left(p, \nabla \cdot \boldsymbol{v}_{1}\right)_{\Omega}+\left(q, \nabla \cdot \boldsymbol{u}_{1}\right)_{\Omega} \\
+\sum_{K \in \mathcal{T}_{h}}\left[-\left(\boldsymbol{u}_{e}, \nu \Delta \boldsymbol{v}_{1}+\nabla q\right)_{K}+\left(\boldsymbol{u}_{e}^{\partial K}, \nu \partial_{\boldsymbol{n}} \boldsymbol{v}_{1}+q \mathbf{I} \cdot \boldsymbol{n}\right)_{\partial K}\right]=\left(\boldsymbol{f}, \boldsymbol{v}_{1}\right)_{\Omega}
\end{gathered}
$$

which, applying characterizations (9)-(10), becomes

$$
\begin{gathered}
\nu\left(\nabla \boldsymbol{u}_{1}, \nabla \boldsymbol{v}_{1}\right)_{\Omega}-\left(p, \nabla \cdot \boldsymbol{v}_{1}\right)_{\Omega}+\left(q, \nabla \cdot \boldsymbol{u}_{1}\right)_{\Omega} \\
+\sum_{K \in \mathcal{T}_{h}}\left[\frac{1}{\nu}\left(\mathcal{M}_{K}\left(-\nu \Delta \boldsymbol{u}_{1}+\nabla p\right)-\mathcal{B}_{K}\left(\llbracket \nu \partial_{\boldsymbol{n}} \boldsymbol{u}_{1}+p \mathbf{I} \cdot \boldsymbol{n} \rrbracket\right), \nu \Delta \boldsymbol{v}_{1}+\nabla q\right)_{K}\right. \\
\left.+\frac{1}{\nu}\left(\mathcal{B}_{K}\left(\llbracket \nu \partial_{\boldsymbol{n}} \boldsymbol{u}_{1}+p \mathbf{I} \cdot \boldsymbol{n} \rrbracket\right), \nu \partial_{\boldsymbol{n}} \boldsymbol{v}_{1}+q \mathbf{I} \cdot \boldsymbol{n}\right)_{\partial K}\right] \\
=\left(\boldsymbol{f}, \boldsymbol{v}_{1}\right)_{\Omega}+\sum_{K \in \mathcal{T}_{h}} \frac{1}{\nu}\left(\mathcal{M}_{K}(\boldsymbol{f}), \nu \Delta \boldsymbol{v}_{1}+\nabla q\right)_{K} .
\end{gathered}
$$

Using this form, in the next sections we will present concrete stabilized finite element methods for both the simplest possible pair ( $\mathbb{P}^{1} / \mathbb{P}^{0}$ elements) and equal order $\mathbb{P}^{1} / \mathbb{P}^{1}$ continuous finite elements.

\section{The simplest element $\mathbb{P}^{1} / \mathbb{P}^{0}$.}

3.1. The method. For this case, the finite element spaces are given by

$$
\mathbf{V}_{h}:=\left\{\boldsymbol{v} \in C^{0}(\bar{\Omega})^{2}:\left.\boldsymbol{v}\right|_{K} \in \mathbb{P}^{1}(K)^{2} \forall K \in \mathcal{T}_{h}\right\} \cap H_{0}^{1}(\Omega)^{2}
$$

for the velocity, and

$$
Q_{h}^{0}:=\left\{q \in L_{0}^{2}(\Omega):\left.q\right|_{K} \in \mathbb{P}^{0}(K) \forall K \in \mathcal{T}_{h}\right\}
$$

for the pressure. Using these spaces, we propose the following stabilized method: Find $\left(\boldsymbol{u}_{1}, p_{0}\right) \in \mathbf{V}_{h} \times Q_{h}^{0}$ such that

$$
\mathbf{B}_{0}\left(\left(\boldsymbol{u}_{1}, p_{0}\right),\left(\boldsymbol{v}_{1}, q_{0}\right)\right)=\mathbf{F}_{0}\left(\boldsymbol{v}_{1}, q_{0}\right) \quad \forall\left(\boldsymbol{v}_{1}, q_{0}\right) \in \mathbf{V}_{h} \times Q_{h}^{0},
$$

where

$$
\begin{gathered}
\mathbf{B}_{0}\left(\left(\boldsymbol{u}_{1}, p_{0}\right),\left(\boldsymbol{v}_{1}, q_{0}\right)\right):=\nu\left(\nabla \boldsymbol{u}_{1}, \nabla \boldsymbol{v}_{1}\right)_{\Omega}-\left(p_{0}, \nabla \cdot \boldsymbol{v}_{1}\right)_{\Omega}+\left(q_{0}, \nabla \cdot \boldsymbol{u}_{1}\right)_{\Omega} \\
+\sum_{Z \in \mathcal{E}_{h}} \tau_{Z}\left(\llbracket \nu \partial_{\boldsymbol{n}} \boldsymbol{u}_{1}+p_{0} \mathbf{I} \cdot \boldsymbol{n} \rrbracket, \llbracket \nu \partial_{\boldsymbol{n}} \boldsymbol{v}_{1}+q_{0} \mathbf{I} \cdot \boldsymbol{n} \rrbracket\right)_{Z}, \\
\mathbf{F}_{0}\left(\boldsymbol{v}_{1}, q_{0}\right):=\left(\boldsymbol{f}, \boldsymbol{v}_{1}\right)_{\Omega},
\end{gathered}
$$

and $\tau_{Z}$ is given by

$$
\tau_{Z}:=\frac{h_{Z}}{12 \nu} .
$$

REMARK 3.1. This method differs somewhat from other existing stabilized finite element methods with discontinuous pressure spaces (see, for example, [23, 29, 34, 14]). 
First, since $\tau_{Z}$ is known exactly, we have no free constants to set. To the authors' knowledge, this is the first time that the stabilization parameter corresponding to jump terms is known exactly. Furthermore, and in contrast to [22], the jump terms are derived without the use of a macroelement technique. Finally, another difference is the nature of the jump terms, not only containing pressure jumps, but also the jump on the normal derivative of $\boldsymbol{u}$.

REMARK 3.2. One of the drawbacks of the RFB method for the Stokes problem is that, due to the zero boundary condition on the element boundary, there is not a bubble-based enrichment that makes stable the $\mathbb{P}^{1} / \mathbb{P}^{0}$ element (see [6] for a discussion), and hence, the use of a different boundary condition makes it possible to stabilize the $\mathbb{P}^{1} / \mathbb{P}^{0}$ element.

3.1.1. Derivation of the method. First we note that, using spaces $\mathbf{V}_{h}$ and $Q_{h}^{0}$, (13) reduces to the following: Find $\left(\boldsymbol{u}_{1}, p_{0}\right) \in \mathbf{V}_{h} \times Q_{h}^{0}$ such that

$$
\begin{aligned}
& \nu\left(\nabla \boldsymbol{u}_{1}, \nabla \boldsymbol{v}_{1}\right)_{\Omega}-\left(p_{0}, \nabla \cdot \boldsymbol{v}_{1}\right)_{\Omega}+\left(q_{0}, \nabla \cdot \boldsymbol{u}_{1}\right)_{\Omega} \\
& +\sum_{Z \in \mathcal{E}_{h}} \frac{1}{\nu}\left(\mathcal{B}_{K}\left(\llbracket \nu \partial_{\boldsymbol{n}} \boldsymbol{u}_{1}+p_{0} \mathbf{I} \cdot \boldsymbol{n} \rrbracket\right), \llbracket \nu \partial_{\boldsymbol{n}} \boldsymbol{v}_{1}+q_{0} \mathbf{I} \cdot \boldsymbol{n} \rrbracket\right)_{Z}=\left(\boldsymbol{f}, \boldsymbol{v}_{1}\right)_{\Omega}
\end{aligned}
$$

for all $\left(\boldsymbol{v}_{1}, q_{0}\right) \in \mathbf{V}_{h} \times Q_{h}^{0}$.

REMARK 3.3. Since $\mathcal{B}_{K}$ is the inverse of an elliptic operator, by denoting $\boldsymbol{v}=$ $\mathcal{B}_{K}(\boldsymbol{g})$, we have, for all $\boldsymbol{g} \in L^{2}(\partial K)^{2}$,

$$
\left(\mathcal{B}_{K}(\boldsymbol{g}), \boldsymbol{g}\right)_{\partial K}=-\left(\boldsymbol{v}, \partial_{\boldsymbol{s s}} \boldsymbol{v}\right)_{\partial K}=\left(\partial_{\boldsymbol{s}} \boldsymbol{v}, \partial_{\boldsymbol{s}} \boldsymbol{v}\right)_{\partial K} \geq 0
$$

and hence we are adding a positive term to the formulation.

Next we exploit the fact that $\llbracket \partial_{\boldsymbol{n}} \boldsymbol{u}_{1}+\left.p_{0} \mathbf{I} \cdot \boldsymbol{n} \rrbracket\right|_{Z}$ is a constant function. To do so, we define the (matrix) function $\boldsymbol{b}_{K}^{u}:=\left(\mathcal{B}_{K}\left(\boldsymbol{e}_{1}\right) \mid \mathcal{B}_{K}\left(\boldsymbol{e}_{2}\right)\right)$, where $\boldsymbol{e}_{1}, \boldsymbol{e}_{2}$ are the canonical vectors in $\mathbb{R}^{2}$, and we remark that, from its definition, $\boldsymbol{b}_{K}^{u}=b_{K}^{u} \mathbf{I}$, where $b_{K}^{u}$ is the solution of

$$
-\Delta b_{K}^{u}=0 \quad \text { in } K, \quad b_{K}^{u}=g(s) \quad \text { on each } Z \subset \partial K,
$$

where $g=0$ if $Z \subset \partial \Omega$, and $g$ satisfies

$$
-\partial_{s s} g(s)=\frac{1}{h_{Z}} \text { in } Z, \quad g=0 \quad \text { at the nodes },
$$

in the internal edges.

REMARK 3.4. The solution of (20) may be calculated explicitly and it is not difficult to realize that

$$
\frac{\left(b_{K}^{u}, 1\right)_{Z}}{|Z|}=\frac{h_{Z}}{12}
$$

Finally, since $\llbracket \partial_{\boldsymbol{n}} \boldsymbol{u}_{1}+\left.p_{0} \mathbf{I} \cdot \boldsymbol{n} \rrbracket\right|_{Z}$ is a constant function we obtain

$$
\begin{aligned}
& \left(\mathcal{B}_{K}\left(\llbracket \nu \partial_{\boldsymbol{n}} \boldsymbol{u}_{1}+p_{0} \mathbf{I} \cdot \boldsymbol{n} \rrbracket\right), \llbracket \nu \partial_{\boldsymbol{n}} \boldsymbol{v}_{1}+q_{0} \mathbf{I} \cdot \boldsymbol{n} \rrbracket\right)_{Z} \\
& =\left[\int_{Z} \boldsymbol{b}_{K}^{u}\right] \llbracket \nu \partial_{\boldsymbol{n}} \boldsymbol{u}_{1}+\left.p_{0} \mathbf{I} \cdot \boldsymbol{n} \rrbracket\right|_{Z} \cdot \llbracket \nu \partial_{\boldsymbol{n}} \boldsymbol{v}_{1}+\left.q_{0} \mathbf{I} \cdot \boldsymbol{n} \rrbracket\right|_{Z} \\
& =\frac{\left(b_{K}^{u}, 1\right)_{Z}}{|Z|}\left(\llbracket \nu \partial_{\boldsymbol{n}} \boldsymbol{u}_{1}+p_{0} \mathbf{I} \cdot \boldsymbol{n} \rrbracket, \llbracket \nu \partial_{\boldsymbol{n}} \boldsymbol{v}_{1}+q_{0} \mathbf{I} \cdot \boldsymbol{n} \rrbracket\right)_{Z},
\end{aligned}
$$


and hence replacing this in (18) and using the previous remark, we obtain method (14).

3.2. Error analysis. From now on, $C$ will denote a positive constant independent of $h$ and $\nu$, and that may change its value whenever it is written in two different places.

The next result states the consistency of the proposed method.

Lemma 3.5. Let $(\boldsymbol{u}, p) \in\left[H^{2}(\Omega) \cap H_{0}^{1}(\Omega)\right]^{2} \times\left[H^{1}(\Omega) \cap L_{0}^{2}(\Omega)\right]$ be the weak solution of (1) and $\left(\boldsymbol{u}_{1}, p_{0}\right)$ the solution of (14). Then,

$$
\mathbf{B}_{0}\left(\left(\boldsymbol{u}-\boldsymbol{u}_{1}, p-p_{0}\right),\left(\boldsymbol{v}_{1}, q_{0}\right)\right)=0 \quad \forall\left(\boldsymbol{v}_{1}, q_{0}\right) \in \mathbf{V}_{h} \times Q_{h}^{0} .
$$

Proof. The results follows by noting that $\llbracket \nu \partial_{\boldsymbol{n}} \boldsymbol{u}+p \mathbf{I} \cdot \boldsymbol{n} \rrbracket=\mathbf{0}$ a.e. across all the internal edges.

Moreover, defining the mesh-dependent norm

$$
\|(\boldsymbol{v}, q)\|_{h}:=\left[\nu|\boldsymbol{v}|_{1, \Omega}^{2}+\sum_{Z \in \mathcal{E}_{h}} \tau_{Z}\left\|\llbracket \nu \partial_{\boldsymbol{n}} \boldsymbol{v}+q \mathbf{I} \cdot \boldsymbol{n} \rrbracket\right\|_{0, Z}^{2}\right]^{\frac{1}{2}},
$$

we have the following continuity and coercivity results.

Lemma 3.6. Let be $(\boldsymbol{v}, q),(\boldsymbol{w}, r) \in\left[H^{2}\left(\mathcal{T}_{h}\right) \cap H_{0}^{1}(\Omega)\right]^{2} \times\left[H^{1}\left(\mathcal{T}_{h}\right) \cap L_{0}^{2}(\Omega)\right]$. Then, bilinear form $\mathbf{B}_{0}$ satisfies

$$
\begin{aligned}
\mathbf{B}_{0}((\boldsymbol{v}, q),(\boldsymbol{w}, r)) & \leq\|(\boldsymbol{v}, q)\|_{h}\|(\boldsymbol{w}, r)\|_{h}+(\nabla \cdot \boldsymbol{v}, r)_{\Omega}-(q, \nabla \cdot \boldsymbol{w})_{\Omega}, \\
\mathbf{B}_{0}((\boldsymbol{v}, q),(\boldsymbol{v}, q)) & =\|(\boldsymbol{v}, q)\|_{h}^{2} .
\end{aligned}
$$

Proof. The result follows immediately from the definition of $\mathbf{B}_{0}$.

In order to perform the numerical analysis of this method, we will consider the Lagrange interpolation operator $I_{h}: C^{0}(\bar{\Omega}) \rightarrow V_{h}$ (if $\boldsymbol{v}=\left(v_{1}, v_{2}\right) \in C^{0}(\bar{\Omega})^{2}$, we denote $\left.I_{h}(\boldsymbol{v})=\left(I_{h}\left(v_{1}\right), I_{h}\left(v_{2}\right)\right)\right)$ to approximate the velocity. Then, it is well known (cf. [17]) that

$$
\begin{gathered}
\left|v-I_{h}(v)\right|_{m, K} \leq C h_{K}^{2-m}|v|_{2, K} \quad \forall v \in H^{2}(K), \\
\left|v-I_{h}(v)\right|_{t, Z} \leq C h_{Z}^{2-t-1 / 2}|v|_{2, \omega_{Z}} \quad \forall v \in H^{2}\left(\omega_{Z}\right)
\end{gathered}
$$

for all $K \in \mathcal{T}_{h}, Z \in \mathcal{E}_{h}$, where $\omega_{Z}:=\cup\left\{K \in \mathcal{T}_{h}: Z \subset \partial K\right\}$, and $m=0,1,2, t=0,1$. Let us remark that to obtain the second estimate above, we used the following local trace theorem (for a proof, see [33]): There exists $C>0$, independent of $h$, such that

$$
\|v\|_{0, \partial K}^{2} \leq C\left(\frac{1}{h_{K}}\|v\|_{0, K}^{2}+h_{K}|v|_{1, K}^{2}\right)
$$

for all $v \in H^{1}(K)$.

In order to approximate the pressure we will consider $\Pi_{h}: L^{2}(\Omega) \rightarrow Q_{h}^{0}$ as the $L^{2}(\Omega)$-projection onto $Q_{h}^{0}$. This projection satisfies (cf. [17])

$$
\left\|q-\Pi_{h}(q)\right\|_{0, \Omega} \leq C h|q|_{1, \Omega}
$$


if $q \in H^{1}(\Omega)$, and hence, using the local trace theorem (28), we obtain

$$
\left[\sum_{Z \in \mathcal{E}_{h}} h_{Z}\left\|\llbracket q-\Pi_{h}(q) \rrbracket\right\|_{0, Z}^{2}\right]^{\frac{1}{2}} \leq C h|q|_{1, \Omega}
$$

for all $q \in H^{1}(\Omega)$.

Lemma 3.7. Suppose $(\boldsymbol{v}, q) \in H^{2}(\Omega)^{2} \times H^{1}(\Omega)$. Then,

$$
\left\|\left(\boldsymbol{v}-I_{h}(\boldsymbol{v}), q-\Pi_{h}(q)\right)\right\|_{h} \leq C h\left(\sqrt{\nu}|\boldsymbol{v}|_{2, \Omega}+\frac{1}{\sqrt{\nu}}|q|_{1, \Omega}\right) .
$$

Proof. The result follows immediately from the norm definition and (26), (27), (30).

Using previous results we can establish the following convergence result.

THEOREM 3.8. Let $(\boldsymbol{u}, p) \in\left[H^{2}(\Omega) \cap H_{0}^{1}(\Omega)\right]^{2} \times\left[H^{1}(\Omega) \cap L_{0}^{2}(\Omega)\right]$ be the solution of (1) and $\left(\boldsymbol{u}_{1}, p_{0}\right)$ the solution of (14). Then, the following error estimate holds:

$$
\left\|\left(\boldsymbol{u}-\boldsymbol{u}_{1}, p-p_{0}\right)\right\|_{h} \leq C h\left(\sqrt{\nu}|\boldsymbol{u}|_{2, \Omega}+\frac{1}{\sqrt{\nu}}|p|_{1, \Omega}\right) .
$$

Proof. Let $\left(\tilde{\boldsymbol{u}}_{h}, \tilde{p}_{h}\right):=\left(I_{h}(\boldsymbol{u}), \Pi_{h}(p)\right) \in \mathbf{V}_{h} \times Q_{h}^{0}$. From Lemmas 3.5 and 3.6 we know that

$$
\begin{aligned}
\left\|\left(\boldsymbol{u}-\boldsymbol{u}_{1}, p-p_{0}\right)\right\|_{h}^{2}= & \mathbf{B}_{0}\left(\left(\boldsymbol{u}-\boldsymbol{u}_{1}, p-p_{0}\right),\left(\boldsymbol{u}-\boldsymbol{u}_{1}, p-p_{0}\right)\right) \\
= & \mathbf{B}_{0}\left(\left(\boldsymbol{u}-\boldsymbol{u}_{1}, p-p_{0}\right),\left(\boldsymbol{u}-\tilde{\boldsymbol{u}}_{h}, p-\tilde{p}_{h}\right)\right) \\
\leq & C\left\|\left(\boldsymbol{u}-\boldsymbol{u}_{1}, p-p_{0}\right)\right\|_{h}\left\|\left(\boldsymbol{u}-\tilde{\boldsymbol{u}}_{h}, p-\tilde{p}_{h}\right)\right\|_{h} \\
& +\left(\nabla \cdot\left(\boldsymbol{u}-\boldsymbol{u}_{1}\right), p-\tilde{p}_{h}\right)_{\Omega}-\left(\nabla \cdot\left(\boldsymbol{u}-\tilde{\boldsymbol{u}}_{h}\right), p-p_{0}\right)_{\Omega} .
\end{aligned}
$$

Now,

$$
\left(\nabla \cdot\left(\boldsymbol{u}-\boldsymbol{u}_{1}\right), p-\tilde{p}_{h}\right)_{\Omega}=-\left(\nabla \cdot \boldsymbol{u}_{1}, p-\tilde{p}_{h}\right)_{\Omega}=0
$$

since $\boldsymbol{u}$ is a solenoidal field and $\nabla \cdot \boldsymbol{u}_{1} \in Q_{h}^{0}$. On the other hand,

$$
\begin{aligned}
& \left(\nabla \cdot\left(\boldsymbol{u}-\tilde{\boldsymbol{u}}_{h}\right), p-p_{0}\right)_{\Omega}=\sum_{K \in \mathcal{T}_{h}}\left[-\left(\nabla p, \boldsymbol{u}-\tilde{\boldsymbol{u}}_{h}\right)_{K}+\left(p-p_{0},\left(\boldsymbol{u}-\tilde{\boldsymbol{u}}_{h}\right) \cdot \boldsymbol{n}\right)_{\partial K}\right] \\
& =-\left(\nabla p, \boldsymbol{u}-\tilde{\boldsymbol{u}}_{h}\right)_{\Omega}+\sum_{K \in \mathcal{T}_{h}}\left(\left(p-p_{0}\right) \mathbf{I} \cdot \boldsymbol{n}, \boldsymbol{u}-\tilde{\boldsymbol{u}}_{h}\right)_{\partial K} \\
& \leq|p|_{1, \Omega}\left\|\boldsymbol{u}-\tilde{\boldsymbol{u}}_{h}\right\|_{0, \Omega}+\sum_{Z \in \mathcal{E}_{h}}\left(\llbracket\left(p-p_{0}\right) \mathbf{I} \cdot \boldsymbol{n} \rrbracket, \boldsymbol{u}-\tilde{\boldsymbol{u}}_{h}\right)_{Z} \\
& \leq C h^{2}|p|_{1, \Omega}|\boldsymbol{u}|_{2, \Omega}+C \sum_{Z \in \mathcal{E}_{h}} \frac{h_{Z}^{\frac{3}{2}}}{\sqrt{\nu}}\left\|\llbracket\left(p-p_{0}\right) \mathbf{I} \cdot \boldsymbol{n} \rrbracket\right\|_{0, Z} \sqrt{\nu}|\boldsymbol{u}|_{2, \omega_{Z}} \\
& \leq C h^{2}|p|_{1, \Omega}|\boldsymbol{u}|_{2, \Omega}+\frac{1}{\gamma} \sum_{Z \in \mathcal{E}_{h}} \frac{h_{Z}}{\nu}\left\|\llbracket\left(p-p_{0}\right) \mathbf{I} \cdot \boldsymbol{n} \rrbracket\right\|_{0, Z}^{2}+C \gamma \sum_{Z \in \mathcal{E}_{h}} h^{2} \nu|\boldsymbol{u}|_{2, \omega_{Z}}^{2} \\
& \leq C h^{2}\left((1+\gamma) \nu|\boldsymbol{u}|_{2, \Omega}^{2}+\frac{1}{\nu}|p|_{1, \Omega}^{2}\right)+\frac{1}{\gamma} \sum_{Z \in \mathcal{E}_{h}} \frac{h_{Z}}{\nu}\left\|\llbracket\left(p-p_{0}\right) \mathbf{I} \cdot \boldsymbol{n} \rrbracket\right\|_{0, Z}^{2},
\end{aligned}
$$


where $\gamma>0$. Now, using the local trace theorem (28) and the fact that $\mathbf{V}_{h}$ is constituted by linear polynomials we arrive at

$$
\begin{aligned}
& \sum_{Z \in \mathcal{E}_{h}} \frac{h_{Z}}{\nu}\left\|\llbracket\left(p-p_{0}\right) \mathbf{I} \cdot \boldsymbol{n} \rrbracket\right\|_{0, Z}^{2} \\
& \leq 2 \sum_{Z \in \mathcal{E}_{h}} \frac{h_{Z}}{\nu}\left(\left\|\llbracket \nu \partial_{\boldsymbol{n}}\left(\boldsymbol{u}-\boldsymbol{u}_{1}\right)+\left(p-p_{0}\right) \mathbf{I} \cdot \boldsymbol{n} \rrbracket\right\|_{0, Z}^{2}+\left\|\llbracket \nu \partial_{\boldsymbol{n}}\left(\boldsymbol{u}-\boldsymbol{u}_{1}\right) \rrbracket\right\|_{0, Z}^{2}\right) \\
& \leq C\left(\sum_{Z \in \mathcal{E}_{h}}\left[\frac{h_{Z}}{\nu}\left\|\llbracket \partial_{\boldsymbol{n}}\left(\boldsymbol{u}-\boldsymbol{u}_{1}\right)+\left(p-p_{0}\right) \mathbf{I} \cdot \boldsymbol{n} \rrbracket\right\|_{0, Z}^{2}\right]+\nu\left|\boldsymbol{u}-\boldsymbol{u}_{1}\right|_{1, \Omega}^{2}+\nu h^{2}|\boldsymbol{u}|_{2, \Omega}^{2}\right) \\
& \leq \tilde{C}\left\|\left(\boldsymbol{u}-\boldsymbol{u}_{1}, p-p_{0}\right)\right\|_{h}^{2}+C \nu h^{2}|\boldsymbol{u}|_{2, \Omega}^{2} .
\end{aligned}
$$

Hence, choosing $\gamma=2 \tilde{C}$ we obtain

$$
\frac{1}{2}\left\|\left(\boldsymbol{u}-\boldsymbol{u}_{1}, p-p_{0}\right)\right\|_{h}^{2} \leq C h^{2}\left(\nu|\boldsymbol{u}|_{2, \Omega}^{2}+\frac{1}{\nu}|p|_{1, \Omega}^{2}\right),
$$

and the result follows by extracting the square root.

REMARK 3.9. The last result gives a convergence result for the velocity, plus a convergence result for the jump terms. More precisely, this result implies $\mid \boldsymbol{u}-$ $\left.\boldsymbol{u}_{1}\right|_{1, \Omega} \leq C h$ and $\left[\sum_{Z \in \mathcal{E}_{h}} h_{Z}\left\|\llbracket \partial_{\boldsymbol{n}}\left(\boldsymbol{u}-\boldsymbol{u}_{1}\right)+\left(p-p_{0}\right) \mathbf{I} \cdot \boldsymbol{n} \rrbracket\right\|_{0, Z}^{2}\right]^{\frac{1}{2}} \leq C h$, which are both optimal in order and regularity.

3.2.1. A convergence result for the pressure. The last result of the previous section does not give convergence on the natural norm of the pressure. That is why a convergence result for the pressure in the $L^{2}(\Omega)$ norm is now given.

In the proof of the next result we will use the Clément interpolation operator (cf. $[17,24]), \mathcal{C}_{h}: H^{1}(\Omega) \rightarrow V_{h}$. This operator satisfies

$$
\left|v-\mathcal{C}_{h}(v)\right|_{m, \Omega} \leq C h^{1-m}|v|_{1, \Omega} \quad \forall v \in H^{1}(\Omega)
$$

for $m=0,1$, with the obvious extension to vector-valued functions.

THEOREM 3.10. Let $(\boldsymbol{u}, p) \in\left[H^{2}(\Omega) \cap H_{0}^{1}(\Omega)\right]^{2} \times\left[H^{1}(\Omega) \cap L_{0}^{2}(\Omega)\right]$ be the solution of (1) and $\left(\boldsymbol{u}_{1}, p_{0}\right)$ the solution of (14). Then, the following error estimate holds:

$$
\left\|p-p_{0}\right\|_{0, \Omega} \leq C h\left[\nu|\boldsymbol{u}|_{2, \Omega}+|p|_{1, \Omega}\right]
$$

Proof. From the continuous inf-sup condition (see [24]), there exists $\boldsymbol{w} \in H_{0}^{1}(\Omega)^{2}$ such that $\nabla \cdot \boldsymbol{w}=p-p_{0}$ in $\Omega$ and $|\boldsymbol{w}|_{1, \Omega} \leq C\left\|p-p_{0}\right\|_{0, \Omega}$. Let $\boldsymbol{w}_{h}=\mathcal{C}_{h}(\boldsymbol{w})$. Then, 
applying the consistency of the method we obtain

$$
\begin{aligned}
& \| p-p_{0} \|_{0, \Omega}^{2}=\left(\nabla \cdot \boldsymbol{w}, p-p_{0}\right)_{\Omega} \\
&=\left(\nabla \cdot\left(\boldsymbol{w}-\boldsymbol{w}_{h}\right), p-p_{0}\right)_{\Omega}+\left(\nabla \cdot \boldsymbol{w}_{h}, p-p_{0}\right)_{\Omega} \\
&= \sum_{K \in \mathcal{T}_{h}}\left[-\left(\boldsymbol{w}-\boldsymbol{w}_{h}, \nabla p\right)_{K}+\left(\boldsymbol{w}-\boldsymbol{w}_{h},\left(p-p_{0}\right) \mathbf{I} \cdot \boldsymbol{n}\right)_{\partial K}\right] \\
&+\nu\left(\nabla\left(\boldsymbol{u}-\boldsymbol{u}_{1}\right), \nabla \boldsymbol{w}_{h}\right)_{\Omega}+\sum_{Z \in \mathcal{E}_{h}} \tau_{Z}\left(\llbracket \nu \partial_{\boldsymbol{n}}\left(\boldsymbol{u}-\boldsymbol{u}_{1}\right)+\left(p-p_{0}\right) \mathbf{I} \cdot \boldsymbol{n} \rrbracket, \llbracket \nu \partial_{\boldsymbol{n}} \boldsymbol{w}_{h} \rrbracket\right)_{Z} \\
& \leq {\left[\sum_{K \in \mathcal{T}_{h}} \frac{h_{K}^{2}}{\nu}|p|_{1, K}^{2}+\sum_{Z \in \mathcal{E}_{h}} \tau_{Z}\left\|\llbracket\left(p-p_{0}\right) \mathbf{I} \cdot \boldsymbol{n} \rrbracket\right\|_{0, Z}^{2}+\nu\left|\boldsymbol{u}-\boldsymbol{u}_{1}\right|_{1, \Omega}^{2}\right.} \\
&\left.+\sum_{Z \in \mathcal{E}_{h}} \tau_{Z}\left\|\llbracket \nu \partial_{\boldsymbol{n}}\left(\boldsymbol{u}-\boldsymbol{u}_{1}\right)+\left(p-p_{0}\right) \mathbf{I} \cdot \boldsymbol{n} \rrbracket\right\|_{0, Z}^{2}\right]^{\frac{1}{2}} \\
& \cdot\left[\sum_{K \in \mathcal{T}_{h}} \frac{\nu}{h_{K}^{2}}\left\|\boldsymbol{w}-\boldsymbol{w}_{h}\right\|_{0, K}^{2}+\sum_{Z \in \mathcal{E}_{h}} \tau_{Z}^{-1}\left\|\boldsymbol{w}-\boldsymbol{w}_{h}\right\|_{0, Z}^{2}\right. \\
&\left.+\nu\left|\boldsymbol{w}_{h}\right|_{1, \Omega}^{2}+\sum_{Z \in \mathcal{E}_{h}} \tau_{Z}\left\|\llbracket \nu \partial_{\boldsymbol{n}} \boldsymbol{w}_{h} \rrbracket\right\|_{0, Z}^{2}\right]^{\frac{1}{2}} .
\end{aligned}
$$

Now, using the local trace theorem (28) and (35) we easily obtain

$$
\begin{gathered}
{\left[\sum_{K \in \mathcal{T}_{h}} \frac{\nu}{h_{K}^{2}}\left\|\boldsymbol{w}-\boldsymbol{w}_{h}\right\|_{0, K}^{2}+\sum_{Z \in \mathcal{E}_{h}} \tau_{Z}^{-1}\left\|\boldsymbol{w}-\boldsymbol{w}_{h}\right\|_{0, Z}^{2}+\nu\left|\boldsymbol{w}_{h}\right|_{1, \Omega}^{2}+\sum_{Z \in \mathcal{E}_{h}} \tau_{Z}\left\|\llbracket \nu \partial_{\boldsymbol{n}} \boldsymbol{w}_{h} \rrbracket\right\|_{0, Z}^{2}\right]^{\frac{1}{2}}} \\
\leq C \sqrt{\nu}|\boldsymbol{w}|_{1, \Omega} \leq C \sqrt{\nu}\left\|p-p_{0}\right\|_{0, \Omega} .
\end{gathered}
$$

Hence, dividing by $\left\|p-p_{0}\right\|_{0, \Omega}$ and using (28) again we have

$$
\begin{aligned}
\| p & -p_{0} \|_{0, \Omega} \\
\leq & C \sqrt{\nu}\left[\sum_{K \in \mathcal{T}_{h}} \frac{h_{K}^{2}}{\nu}|p|_{1, K}^{2}+\nu\left|\boldsymbol{u}-\boldsymbol{u}_{1}\right|_{1, \Omega}^{2}\right. \\
& \left.+\sum_{Z \in \mathcal{E}_{h}} \tau_{Z}\left\|\llbracket \nu \partial_{\boldsymbol{n}}\left(\boldsymbol{u}-\boldsymbol{u}_{1}\right)+\left(p-p_{0}\right) \mathbf{I} \cdot \boldsymbol{n} \rrbracket\right\|_{0, Z}^{2}+\tau_{Z}\left\|\llbracket \nu \partial_{\boldsymbol{n}}\left(\boldsymbol{u}-\boldsymbol{u}_{1}\right) \rrbracket\right\|_{0, Z}^{2}\right]^{\frac{1}{2}} \\
\leq & C \sqrt{\nu}\left[\frac{h^{2}}{\nu}|p|_{1, \Omega}^{2}+\left\|\left(\boldsymbol{u}-\boldsymbol{u}_{1}, p-p_{0}\right)\right\|_{h}^{2}+\nu h^{2}|\boldsymbol{u}|_{2, \Omega}^{2}\right]^{\frac{1}{2}} \\
\leq & C \sqrt{\nu}\left[\frac{h^{2}}{\nu}|p|_{1, \Omega}^{2}+\nu h^{2}|\boldsymbol{u}|_{2, \Omega}^{2}\right]^{\frac{1}{2}}
\end{aligned}
$$

and the result follows.

3.2.2. An error estimate for $\left\|\boldsymbol{u}-\boldsymbol{u}_{\mathbf{1}}\right\|_{0, \Omega}$. Throughout this section we will assume that the solution of the following problem, where $\left(\boldsymbol{u}_{1}, p_{0}\right)$ is the solution of 
(14), belongs to $\left[H^{2}(\Omega) \cap H_{0}^{1}(\Omega)\right]^{2} \times\left[H^{1}(\Omega) \cap L_{0}^{2}(\Omega)\right]$ : Find $(\varphi, \pi)$ such that

$$
\begin{aligned}
-\nu \Delta \varphi-\nabla \pi=\boldsymbol{u}-\boldsymbol{u}_{1}, & \nabla \cdot \boldsymbol{\varphi}=0 \quad \text { in } \Omega, \\
\boldsymbol{\varphi}=\mathbf{0} & \text { on } \partial \Omega .
\end{aligned}
$$

We also assume that the following estimate holds:

$$
\nu\|\boldsymbol{\varphi}\|_{2, \Omega}+\|\pi\|_{1, \Omega} \leq C\left\|\boldsymbol{u}-\boldsymbol{u}_{1}\right\|_{0, \Omega} .
$$

THEOREM 3.11. Let $(\boldsymbol{u}, p) \in\left[H^{2}(\Omega) \cap H_{0}^{1}(\Omega)\right]^{2} \times\left[H^{1}(\Omega) \cap L_{0}^{2}(\Omega)\right]$ be the solution of (1) and $\left(\boldsymbol{u}_{1}, p_{0}\right)$ the solution of (14). Then, the following error estimate holds:

$$
\left\|\boldsymbol{u}-\boldsymbol{u}_{1}\right\|_{0, \Omega} \leq C h^{2}\left(|\boldsymbol{u}|_{2, \Omega}+\frac{1}{\nu}|p|_{1, \Omega}\right) .
$$

Proof. Let $\left(\boldsymbol{\varphi}_{h}, \pi_{h}\right):=\left(I_{h}(\boldsymbol{\varphi}), \Pi_{h}(\pi)\right) \in \mathbf{V}_{h} \times Q_{h}^{0}$. Then, multiplying the first equation in (37) by $\boldsymbol{u}-\boldsymbol{u}_{1}$ and the second by $-\left(p-p_{0}\right)$, from the definition of bilinear form $\mathbf{B}_{0}$, the regularity of $(\boldsymbol{\varphi}, \pi)$ and the consistency of the method and Lemma 3.6, we obtain

$$
\begin{aligned}
\left\|\boldsymbol{u}-\boldsymbol{u}_{1}\right\|_{0, \Omega}^{2}= & \nu\left(\nabla \boldsymbol{\varphi}, \nabla\left(\boldsymbol{u}-\boldsymbol{u}_{1}\right)\right)_{\Omega}+\left(\pi, \nabla \cdot\left(\boldsymbol{u}-\boldsymbol{u}_{1}\right)\right)_{\Omega}-\left(p-p_{0}, \nabla \cdot \boldsymbol{\varphi}\right)_{\Omega} \\
= & \mathbf{B}_{0}\left(\left(\boldsymbol{u}-\boldsymbol{u}_{1}, p-p_{0}\right),(\boldsymbol{\varphi}, \pi)\right) \\
= & \mathbf{B}_{0}\left(\left(\boldsymbol{u}-\boldsymbol{u}_{1}, p-p_{0}\right),\left(\boldsymbol{\varphi}-\boldsymbol{\varphi}_{h}, \pi-\pi_{h}\right)\right) \\
\leq & \left\|\left(\boldsymbol{u}-\boldsymbol{u}_{1}, p-p_{0}\right)\right\|_{h}\left\|\left(\boldsymbol{\varphi}-\boldsymbol{\varphi}_{h}, \pi-\pi_{h}\right)\right\|_{h} \\
& -\left(p-p_{0}, \nabla \cdot\left(\boldsymbol{\varphi}-\boldsymbol{\varphi}_{h}\right)\right)_{\Omega}+\left(\pi-\pi_{h}, \nabla \cdot\left(\boldsymbol{u}-\boldsymbol{u}_{1}\right)\right)_{\Omega} .
\end{aligned}
$$

Now, using (33) we see that $\left(\pi-\pi_{h}, \nabla \cdot\left(\boldsymbol{u}-\boldsymbol{u}_{1}\right)\right)_{\Omega}=0$, and hence, using interpolation inequalities (26), Lemma 3.7 and Theorems 3.8 and 3.10, we arrive at

$$
\begin{aligned}
& \left\|\boldsymbol{u}-\boldsymbol{u}_{1}\right\|_{0, \Omega}^{2} \\
& \leq\left\|\left(\boldsymbol{u}-\boldsymbol{u}_{1}, p-p_{0}\right)\right\|_{h}\left\|\left(\boldsymbol{\varphi}-\boldsymbol{\varphi}_{h}, \pi-\pi_{h}\right)\right\|_{h}+\left\|p-p_{0}\right\|_{0, \Omega}\left\|\nabla \cdot\left(\boldsymbol{\varphi}-\boldsymbol{\varphi}_{h}\right)\right\|_{0, \Omega} \\
& \leq\left[\left\|\left(\boldsymbol{u}-\boldsymbol{u}_{1}, p-p_{0}\right)\right\|_{h}^{2}+\frac{1}{\nu}\left\|p-p_{0}\right\|_{0, \Omega}^{2}\right]^{\frac{1}{2}}\left[\left\|\left(\boldsymbol{\varphi}-\boldsymbol{\varphi}_{h}, \pi-\pi_{h}\right)\right\|_{h}^{2}+\nu\left\|\nabla \cdot\left(\boldsymbol{\varphi}-\boldsymbol{\varphi}_{h}\right)\right\|_{0, \Omega}^{2}\right]^{\frac{1}{2}} \\
& \leq C h^{2}\left[\nu|\boldsymbol{u}|_{2, \Omega}^{2}+\frac{1}{\nu}|p|_{1, \Omega}^{2}\right]^{\frac{1}{2}}\left[\nu|\boldsymbol{\varphi}|_{2, \Omega}^{2}+\frac{1}{\nu}|\pi|_{1, \Omega}^{2}\right]^{\frac{1}{2}} \\
& \leq C \frac{1}{\sqrt{\nu}} h^{2}\left(\sqrt{\nu}|\boldsymbol{u}|_{2, \Omega}+\frac{1}{\sqrt{\nu}}|p|_{1, \Omega}\right)\left\|\boldsymbol{u}-\boldsymbol{u}_{1}\right\|_{0, \Omega},
\end{aligned}
$$

and the result follows.

\section{The method using $\mathbb{P}^{1} / \mathbb{P}^{1}$ continuous elements.}

4.1. The method. For this case, the finite element space for the velocity is the same as in previous section, but the pressure space is now given by

$$
Q_{h}^{1}:=\left\{q \in C^{0}(\bar{\Omega}):\left.q\right|_{K} \in \mathbb{P}^{1}(K) \forall K \in \mathcal{T}_{h}\right\} \cap L_{0}^{2}(\Omega) .
$$

As we will see in next section, the method coming directly from (13) is given by the following: Find $\left(\tilde{\boldsymbol{u}}_{1}, \tilde{p}_{1}\right) \in \mathbf{V}_{h} \times Q_{h}^{1}$ such that

$$
\mathbf{B}_{1}\left(\left(\tilde{\boldsymbol{u}}_{1}, \tilde{p}_{1}\right),\left(\boldsymbol{v}_{1}, q_{1}\right)\right)=\mathbf{F}\left(\boldsymbol{v}_{1}, q_{1}\right) \quad \forall\left(\boldsymbol{v}_{1}, q_{1}\right) \in \mathbf{V}_{h} \times Q_{h}^{1},
$$


where

$$
\mathbf{B}_{1}\left(\left(\boldsymbol{u}_{1}, p_{1}\right),\left(\boldsymbol{v}_{1}, q_{1}\right)\right):=\mathbf{B}\left(\left(\boldsymbol{u}_{1}, p_{1}\right),\left(\boldsymbol{v}_{1}, q_{1}\right)\right)-\sum_{K \in \mathcal{T}_{h}} \frac{1}{\nu}\left(\mathcal{B}_{K}\left(\llbracket \nu \partial_{\boldsymbol{n}} \boldsymbol{u}_{1} \rrbracket\right), \nabla q_{1}\right)_{K}
$$

with

$$
\begin{gathered}
\mathbf{B}\left(\left(\boldsymbol{u}_{1}, p_{1}\right),\left(\boldsymbol{v}_{1}, q_{1}\right)\right):=\nu\left(\nabla \boldsymbol{u}_{1}, \nabla \boldsymbol{v}_{1}\right)_{\Omega}-\left(p_{1}, \nabla \cdot \boldsymbol{v}_{1}\right)_{\Omega}+\left(q_{1}, \nabla \cdot \boldsymbol{u}_{1}\right)_{\Omega} \\
+\sum_{K \in \mathcal{T}_{h}} \tau_{K}\left(-\nu \Delta \boldsymbol{u}_{1}+\nabla p_{1}, \nu \Delta \boldsymbol{v}_{1}+\nabla q_{1}\right)_{K}+\sum_{Z \in \mathcal{E}_{h}} \tau_{Z}\left(\llbracket \nu \partial_{\boldsymbol{n}} \boldsymbol{u}_{1} \rrbracket, \llbracket \nu \partial_{\boldsymbol{n}} \boldsymbol{v}_{1} \rrbracket\right)_{Z}, \\
\mathbf{F}\left(\boldsymbol{v}_{1}, q_{1}\right):=\left(\boldsymbol{f}, \boldsymbol{v}_{1}\right)_{\Omega}+\sum_{K \in \mathcal{T}_{h}} \tau_{K}\left(\boldsymbol{f}, \nu \Delta \boldsymbol{v}_{1}+\nabla q_{1}\right)_{K}, \\
\tau_{K}:=C_{1} \frac{h_{K}^{2}}{\nu},
\end{gathered}
$$

where $\tau_{Z}$ is given by (17) and $C_{1}=\frac{1}{8}$. The value $C_{1}=\frac{1}{8}$ has been suggested by the error analysis of original method (39) (see Appendix A).

Now, for reasons that we will justify later (see Theorem 4.3 below), we will drop the term

$$
-\sum_{K \in \mathcal{T}_{h}}\left(\frac{1}{\nu} \mathcal{B}_{K}\left(\llbracket \nu \partial_{\boldsymbol{n}} \boldsymbol{u}_{1} \rrbracket\right), \nabla q_{1}\right)_{K}
$$

and analyze (and implement) the following simplified version of (39): Find $\left(\boldsymbol{u}_{1}, p_{1}\right) \in$ $\mathbf{V}_{h} \times Q_{h}^{1}$ such that

$$
\mathbf{B}\left(\left(\boldsymbol{u}_{1}, p_{1}\right),\left(\boldsymbol{v}_{1}, q_{1}\right)\right)=\mathbf{F}\left(\boldsymbol{v}_{1}, q_{1}\right) \quad \forall\left(\boldsymbol{v}_{1}, q_{1}\right) \in \mathbf{V}_{h} \times Q_{h}^{1}
$$

REMARK 4.1. We see that method (44) has the form of a stabilized method of the GLS class, plus a nonstandard jump term formed by the residual of the Cauchy stress tensor on the edges of the triangulation. This will give us control of this residual, which is exclusive to continuous pressure spaces, since in that case pressure jumps vanish.

REMARK 4.2. The method is written as the restriction of a consistent method to $\mathbb{P}^{1} / \mathbb{P}^{1}$ elements simply to avoid some technical difficulties. A nonconsistent presentation may be given and in that case we can prove that the consistency error does not imply a loss of precision.

As we said before, we will perform the error analysis of method (44). This is due to the fact that the error of method (39) is bounded by that of (44), as stated in the following result, whose proof may be found in Appendix A.

THEOREM 4.3. Let $(\boldsymbol{u}, p) \in H^{2}(\Omega)^{2} \times H^{1}(\Omega)$ be the solution of (1). Then, method (39) is consistent. Moreover, (39) has a unique solution $\left(\tilde{\boldsymbol{u}}_{1}, \tilde{p}_{1}\right) \in \mathbf{V}_{h} \times Q_{h}^{1}$, and the following error estimate holds:

$$
\left\|\boldsymbol{u}-\tilde{\boldsymbol{u}}_{1}\right\|_{h}^{2}+\left\|p-\tilde{p}_{1}\right\|_{h}^{2} \leq C\left(\left\|\boldsymbol{u}-\boldsymbol{u}_{1}\right\|_{h}^{2}+\left\|p-p_{1}\right\|_{h}^{2}\right),
$$

where $\left(\boldsymbol{u}_{1}, p_{1}\right) \in \mathbf{V}_{h} \times Q_{h}^{1}$ is the solution of (44), and the norms are defined as in (49)-(50) below. 
4.1.1. Derivation of the method. Using spaces $\mathbf{V}_{h}$ and $Q_{h}^{1}$, (13) reduces to the following: Find $\left(\boldsymbol{u}_{1}, p_{1}\right) \in \mathbf{V}_{h} \times Q_{h}^{1}$ such that

$$
\begin{aligned}
& \nu\left(\nabla \boldsymbol{u}_{1}, \nabla \boldsymbol{v}_{1}\right)_{\Omega}-\left(p_{1}, \nabla \cdot \boldsymbol{v}_{1}\right)_{\Omega}+\left(q_{1}, \nabla \cdot \boldsymbol{u}_{1}\right)_{\Omega} \\
& +\sum_{K \in \mathcal{T}_{h}} \frac{1}{\nu}\left(\mathcal{M}_{K}\left(\nabla p_{1}\right)-\mathcal{B}_{K}\left(\llbracket \nu \partial_{\boldsymbol{n}} \boldsymbol{u}_{1} \rrbracket\right), \nabla q_{1}\right)_{K} \\
(45)+ & \sum_{Z \in \mathcal{E}_{h}} \frac{1}{\nu}\left(\mathcal{B}_{K}\left(\llbracket \nu \partial_{\boldsymbol{n}} \boldsymbol{u}_{1} \rrbracket\right), \llbracket \nu \partial_{\boldsymbol{n}} \boldsymbol{v}_{1} \rrbracket\right)_{Z}=\left(\boldsymbol{f}, \boldsymbol{v}_{1}\right)_{\Omega}+\sum_{K \in \mathcal{T}_{h}} \frac{1}{\nu}\left(\mathcal{M}_{K}(\boldsymbol{f}), \nabla q_{1}\right)_{K}
\end{aligned}
$$

for all $\left(\boldsymbol{v}_{1}, q_{1}\right) \in \mathbf{V}_{h} \times Q_{h}^{1}$. Since $\left.\nabla p_{1}\right|_{K} \in \mathbb{R}^{2}$, we have

$$
\mathcal{M}_{K}\left(\nabla p_{1}\right)=\left(\mathcal{M}_{K}\left(\boldsymbol{e}_{1}\right), \mathcal{M}_{K}\left(\boldsymbol{e}_{2}\right)\right) \nabla p_{1}=: \boldsymbol{b}_{K}^{p} \nabla p_{1} \text {. }
$$

As in the previous section, we see that $\boldsymbol{b}_{K}^{p}=b_{K}^{p} \mathbf{I}$, where $b_{K}^{p}$ is the solution of

$$
-\Delta b_{K}^{p}=1 \quad \text { in } K, \quad b_{K}^{p}=0 \quad \text { on } \partial K .
$$

Hence

$$
\left(\mathcal{M}_{K}\left(\nabla p_{1}\right), \nabla q_{1}\right)_{K}=\left.\left.\left[\int_{K} b_{K}^{p}\right] \nabla p_{1}\right|_{K} \cdot \nabla q_{1}\right|_{K}=\frac{\left(b_{K}^{p}, 1\right)_{K}}{|K|}\left(\nabla p_{1}, \nabla q_{1}\right)_{K} .
$$

On the other hand, from the previous section we know that

$$
\left(\mathcal{B}_{K}\left(\llbracket \nu \partial_{\boldsymbol{n}} \boldsymbol{u}_{1} \rrbracket\right), \llbracket \nu \partial_{\boldsymbol{n}} \boldsymbol{v}_{1} \rrbracket\right)_{Z}=\tau_{Z}\left(\llbracket \nu \partial_{\boldsymbol{n}} \boldsymbol{u}_{1} \rrbracket, \llbracket \nu \partial_{\boldsymbol{n}} \boldsymbol{v}_{1} \rrbracket\right)_{Z},
$$

where $\tau_{Z}$ has been defined in (17). Moreover, if we suppose that $f$ is piecewise constant, we have $\mathcal{M}_{K}(\boldsymbol{f})=b_{K}^{p} \boldsymbol{f}$, and hence, in the same way as before,

$$
\left(\mathcal{M}_{K}(\boldsymbol{f}), \nabla q_{1}\right)_{K}=\frac{\left(b_{K}^{p}, 1\right)_{K}}{|K|}\left(\boldsymbol{f}, \nabla q_{1}\right)_{K} .
$$

Summing all this up, we arrive at the following expression for (45): Find $\left(\boldsymbol{u}_{1}, p_{1}\right) \in$ $\mathbf{V}_{h} \times Q_{h}^{1}$ such that

$$
\begin{gathered}
\nu\left(\nabla \boldsymbol{u}_{1}, \nabla \boldsymbol{v}_{1}\right)_{\Omega}-\left(p_{1}, \nabla \cdot \boldsymbol{v}_{1}\right)_{\Omega}+\left(q_{1}, \nabla \cdot \boldsymbol{u}_{1}\right)_{\Omega}+\sum_{K \in \mathcal{T}_{h}} \frac{\left(b_{K}^{p}, 1\right)_{K}}{|K| \nu}\left(\nabla p_{1}, \nabla q_{1}\right)_{K} \\
-\sum_{K \in \mathcal{T}_{h}} \frac{1}{\nu}\left(\mathcal{B}_{K}\left(\llbracket \nu \partial_{\boldsymbol{n}} \boldsymbol{u}_{1} \rrbracket\right), \nabla q_{1}\right)_{K}+\sum_{Z \in \mathcal{E}_{h}} \frac{\left(b_{K}^{u}, 1\right)_{Z}}{|Z| \nu}\left(\llbracket \nu \partial_{\boldsymbol{n}} \boldsymbol{u}_{1} \rrbracket, \llbracket \nu \partial_{\boldsymbol{n}} \boldsymbol{v}_{1} \rrbracket\right)_{Z} \\
=\left(\boldsymbol{f}, \boldsymbol{v}_{1}\right)_{\Omega}+\sum_{K \in \mathcal{T}_{h}} \frac{\left(b_{K}^{p}, 1\right)_{K}}{|K| \nu}\left(\boldsymbol{f}, \nabla q_{1}\right)_{K}
\end{gathered}
$$

for all $\left(\boldsymbol{v}_{1}, q_{1}\right) \in \mathbf{V}_{h} \times Q_{h}^{1}$. Finally, since the mesh is regular by a scaling argument (cf. [31]) we have that

$$
\frac{1}{|K|}\left(b_{K}^{p}, 1\right)_{K} \sim C_{1} h_{K}^{2},
$$

where $C_{1}$ is a positive constant independent of $h$ and $\nu$. Hence, replacing (48) in (47) and defining $\tau_{K}$ appropriately, we obtain method (39).

REMARK 4.4. The assumption of the piecewise constant $\boldsymbol{f}$ on the right-hand side is made simply to derive the method, but it does not affect the precision of it. Indeed, if we consider a general $f \in H^{1}(\Omega)^{2}$ and take its projection onto the space of piecewise constant functions, we keep the same order of convergence of the method (see Appendix B). 
4.2. Error analysis. Let us consider the mesh-dependent norms

$$
\begin{gathered}
\|\boldsymbol{v}\|_{h}^{2}:=\nu|\boldsymbol{v}|_{1, \Omega}^{2}+\sum_{Z \in \mathcal{E}_{h}} \tau_{Z}\left\|\llbracket \nu \partial_{\boldsymbol{n}} \boldsymbol{v} \rrbracket\right\|_{0, Z}^{2}, \\
\|q\|_{h}^{2}:=\sum_{K \in \mathcal{T}_{h}} \tau_{K}|q|_{1, K}^{2} .
\end{gathered}
$$

The first results concern the consistency and well-posedness of stabilized method (44).

LEMma 4.5. Let $(\boldsymbol{u}, p) \in\left[H^{2}(\Omega) \cap H_{0}^{1}(\Omega)\right]^{2} \times\left[H^{1}(\Omega) \cap L_{0}^{2}(\Omega)\right]$ be the solution of (1) and $\left(\boldsymbol{u}_{1}, p_{1}\right)$ the solution of (44). Then,

$$
\mathbf{B}\left(\left(\boldsymbol{u}-\boldsymbol{u}_{1}, p-p_{1}\right),\left(\boldsymbol{v}_{1}, q_{1}\right)\right)=0 \quad \forall\left(\boldsymbol{v}_{1}, q_{1}\right) \in \mathbf{V}_{h} \times Q_{h}^{1} .
$$

Proof. The result follows from the definition of $\mathbf{B}$ and the fact that $\llbracket \nu \partial_{\boldsymbol{n}} \boldsymbol{u} \rrbracket=\mathbf{0}$ a.e. on the internal edges.

Lemma 4.6. Let be $\left(\boldsymbol{v}_{1}, q_{1}\right) \in \mathbf{V}_{h} \times Q_{h}^{1}$. Then

$$
\mathbf{B}\left(\left(\boldsymbol{v}_{1}, q_{1}\right),\left(\boldsymbol{v}_{1}, q_{1}\right)\right)=\left\|\boldsymbol{v}_{1}\right\|_{h}^{2}+\left\|q_{1}\right\|_{h}^{2} .
$$

Proof. The result follows from the definition of $\mathbf{B}$ and the fact that $\Delta \boldsymbol{v}_{1}=\mathbf{0}$ in each $K \in \mathcal{T}_{h}$.

Now, in order to approximate the velocity we will consider the Lagrange interpolation operator as in the previous section and for the pressure interpolation we will use the Clément interpolation operator $\mathcal{C}_{h}$ satisfying (35).

The following approximation result will be useful in what follows.

Lemma 4.7. Let $(\boldsymbol{v}, q) \in\left[H^{2}(\Omega) \cap H_{0}^{1}(\Omega)\right]^{2} \times\left[H^{1}(\Omega) \cap L_{0}^{2}(\Omega)\right]$ and $\tilde{q}_{h}:=\mathcal{C}_{h}(q)-$ $\frac{\left(\mathcal{C}_{h}(q), 1\right)_{\Omega}}{|\Omega|}$. Then,

$$
\begin{gathered}
\left\|\boldsymbol{v}-I_{h}(\boldsymbol{v})\right\|_{h}^{2}+\sum_{K \in \mathcal{T}_{h}}\left[\tau_{K}^{-1}\left\|\boldsymbol{v}-I_{h}(\boldsymbol{v})\right\|_{0, K}^{2}+\nu h_{K}^{2}\left\|\Delta\left(\boldsymbol{v}-I_{h}(\boldsymbol{v})\right)\right\|_{0, K}^{2}\right] \leq C h^{2} \nu|\boldsymbol{v}|_{2, \Omega}^{2}, \\
\left\|q-\tilde{q}_{h}\right\|_{h}+\frac{1}{\sqrt{\nu}}\left\|q-\tilde{q}_{h}\right\|_{0, \Omega} \leq C \frac{h}{\sqrt{\nu}}|q|_{1, \Omega} .
\end{gathered}
$$

Proof. The result follows from the norm definition and using $\left\|q-\tilde{q}_{h}\right\|_{0, \Omega} \leq$ $\left\|q-\mathcal{C}_{h}(q)\right\|_{0, \Omega}$ combined with (26), (27), and (35).

Using Lemmas 4.5-4.7 we can establish the following convergence result.

THEOREM 4.8. Let $(\boldsymbol{u}, p) \in\left[H^{2}(\Omega) \cap H_{0}^{1}(\Omega)\right]^{2} \times\left[H^{1}(\Omega) \cap L_{0}^{2}(\Omega)\right]$ be the solution of (1) and $\left(\boldsymbol{u}_{1}, p_{1}\right)$ the solution of (44). Then, the following error estimate holds:

$$
\left\|\boldsymbol{u}-\boldsymbol{u}_{1}\right\|_{h}+\left\|p-p_{1}\right\|_{h} \leq C h\left[\sqrt{\nu}|\boldsymbol{u}|_{2, \Omega}+\frac{1}{\sqrt{\nu}}|p|_{1, \Omega}\right]
$$

Proof. Let $\tilde{\boldsymbol{u}}_{h}:=I_{h}(\boldsymbol{u}), \tilde{p}_{h}:=\mathcal{C}_{h}(p)-\frac{\left(\mathcal{C}_{h}(p), 1\right)_{\Omega}}{|\Omega|}$ and $\left(\eta^{\boldsymbol{u}}, \eta^{p}\right):=\left(\boldsymbol{u}-\tilde{\boldsymbol{u}}_{h}, p-\tilde{p}_{h}\right)$. Applying Lemma 4.6 and the consistency of the method, and integrating by parts we 
have

$$
\begin{aligned}
&\left\|\boldsymbol{u}_{1}-\tilde{\boldsymbol{u}}_{h}\right\|_{h}^{2}+\left\|p_{1}-\tilde{p}_{h}\right\|_{h}^{2}=\mathbf{B}\left(\left(\boldsymbol{u}_{1}-\tilde{\boldsymbol{u}}_{h}, p_{1}-\tilde{p}_{h}\right),\left(\boldsymbol{u}_{1}-\tilde{\boldsymbol{u}}_{h}, p_{1}-\tilde{p}_{h}\right)\right) \\
&=\mathbf{B}\left(\left(\eta^{\boldsymbol{u}}, \eta^{p}\right),\left(\boldsymbol{u}_{1}-\tilde{\boldsymbol{u}}_{h}, p_{1}-\tilde{p}_{h}\right)\right) \\
&= \nu\left(\nabla \eta^{\boldsymbol{u}}, \nabla\left(\boldsymbol{u}_{1}-\tilde{\boldsymbol{u}}_{h}\right)\right)_{\Omega}-\left(\eta^{p}, \nabla \cdot\left(\boldsymbol{u}_{1}-\tilde{\boldsymbol{u}}_{h}\right)\right)_{\Omega}-\left(\eta^{\boldsymbol{u}}, \nabla\left(p_{1}-\tilde{p}_{h}\right)\right)_{\Omega} \\
&+\sum_{K \in \mathcal{T}_{h}} \tau_{K}\left(-\nu \Delta \eta^{\boldsymbol{u}}, \nabla\left(p_{1}-\tilde{p}_{h}\right)\right)_{K}+\sum_{K \in \mathcal{T}_{h}} \tau_{K}\left(\nabla \eta^{p}, \nabla\left(p_{1}-\tilde{p}_{h}\right)\right)_{K} \\
&+\sum_{Z \in \mathcal{E}_{h}} \tau_{Z}\left(\llbracket \nu \partial_{\boldsymbol{n}} \eta^{\boldsymbol{u}} \rrbracket, \llbracket \nu \partial_{\boldsymbol{n}}\left(\boldsymbol{u}_{1}-\tilde{\boldsymbol{u}}_{h}\right) \rrbracket\right)_{Z} \\
& \leq {\left[\nu\left|\eta^{\boldsymbol{u}}\right|_{1, \Omega}^{2}+\frac{1}{\nu}\left\|\eta^{p}\right\|_{0, \Omega}^{2}+\sum_{K \in \mathcal{T}_{h}}\left(\tau_{K}^{-1}\left\|\eta^{\boldsymbol{u}}\right\|_{0, K}^{2}+\nu^{2} \tau_{K}\left\|\Delta \eta^{\boldsymbol{u}}\right\|_{0, K}^{2}\right)\right.} \\
&\left.+\left\|\eta^{p}\right\|_{h}^{2}+\sum_{Z \in \mathcal{E}_{h}} \tau_{Z}\left\|\llbracket \nu \partial_{\boldsymbol{n}} \eta^{\boldsymbol{u}} \rrbracket\right\|_{0, Z}^{2}\right]^{\frac{1}{2}} \\
& \cdot\left[3 \nu\left|\boldsymbol{u}_{1}-\tilde{\boldsymbol{u}}_{h}\right|_{1, \Omega}^{2}+3 \sum_{K \in \mathcal{T}_{h}} \tau_{K}\left\|\nabla\left(p_{1}-\tilde{p}_{h}\right)\right\|_{0, K}^{2}+\sum_{Z \in \mathcal{E}_{h}} \tau_{Z}\left\|\llbracket \nu \partial_{\boldsymbol{n}}\left(\boldsymbol{u}_{1}-\tilde{\boldsymbol{u}}_{h}\right) \rrbracket\right\|_{0, Z}^{2}\right]^{\frac{1}{2}} \\
& \leq \sqrt{3}\left[\left\|\eta^{\boldsymbol{u}}\right\|_{h}^{2}+\sum_{K \in \mathcal{T}_{h}}\left[\tau_{K}^{-1}\left\|\eta^{\boldsymbol{u}}\right\|_{0, K}^{2}+\nu h_{K}^{2}\left\|\Delta \eta^{\boldsymbol{u}}\right\|_{0, K}^{2}\right]+\left\|\eta^{p}\right\|_{h}^{2}+\frac{1}{\nu}\left\|\eta^{p}\right\|_{0, \Omega}^{2}\right]^{\frac{1}{2}} \\
& \cdot\left[\left\|\boldsymbol{u}_{1}-\tilde{\boldsymbol{u}}_{h}\right\|_{h}^{2}+\left\|p_{1}-\tilde{p}_{h}\right\|_{h}^{2}\right]^{\frac{1}{2}} .
\end{aligned}
$$

Hence, dividing by the last term and applying Lemma 4.7 we arrive at

$$
\left\|\boldsymbol{u}_{1}-\tilde{\boldsymbol{u}}_{h}\right\|_{h}+\left\|p_{1}-\tilde{p}_{h}\right\|_{h} \leq C\left[\nu h^{2}|\boldsymbol{u}|_{2, \Omega}^{2}+\frac{h^{2}}{\nu}|p|_{1, \Omega}^{2}\right]^{\frac{1}{2}}
$$

The result follows using triangular inequality and Lemma 4.7 once more.

REMARK 4.9. In particular, from the previous theorem we have an $O(h)$ convergence for $\left|\boldsymbol{u}-\boldsymbol{u}_{1}\right|_{1, \Omega}$ and $\left[\sum_{Z \in \mathcal{E}_{h}} h_{Z}\left\|\llbracket \partial_{\boldsymbol{n}}\left(\boldsymbol{u}-\boldsymbol{u}_{1}\right) \rrbracket\right\|_{0, Z}^{2}\right]^{\frac{1}{2}}$, which are both optimal in order and regularity.

4.2.1. A convergence result for the pressure. In the last result of the previous section we had an error estimate in the velocity, but, due to the norm definition, we did not guarantee the convergence of the pressure. The next result shows that we have an optimal error estimate in the natural norm of the pressure, which is independent of $\nu$.

THEOREM 4.10. Let $(\boldsymbol{u}, p) \in\left[H^{2}(\Omega) \cap H_{0}^{1}(\Omega)\right]^{2} \times\left[H^{1}(\Omega) \cap L_{0}^{2}(\Omega)\right]$ be the solution of (1) and $\left(\boldsymbol{u}_{1}, p_{1}\right)$ the solution of (44). Then, the following error estimate holds:

$$
\left\|p-p_{1}\right\|_{0, \Omega} \leq C h\left[\nu|\boldsymbol{u}|_{2, \Omega}+|p|_{1, \Omega}\right] .
$$

Proof. From the continuous inf-sup condition (see [24]), there exists $\boldsymbol{w} \in H_{0}^{1}(\Omega)^{2}$ such that $\nabla \cdot \boldsymbol{w}=p-p_{1}$ in $\Omega$ and $|\boldsymbol{w}|_{1, \Omega} \leq C\left\|p-p_{1}\right\|_{0, \Omega}$. Let $\boldsymbol{w}_{h}=\mathcal{C}_{h}(\boldsymbol{w}) \in \mathbf{V}_{h}$. 
Then, applying the consistency of the method, (35), and previous theorem, we obtain

$$
\begin{aligned}
\left\|p-p_{1}\right\|_{0, \Omega}^{2}= & \left(\nabla \cdot \boldsymbol{w}, p-p_{1}\right)_{\Omega}=\left(\nabla \cdot\left(\boldsymbol{w}-\boldsymbol{w}_{h}\right), p-p_{1}\right)_{\Omega}+\left(\nabla \cdot \boldsymbol{w}_{h}, p-p_{1}\right)_{\Omega} \\
= & -\sum_{K \in \mathcal{T}_{h}}\left(\boldsymbol{w}-\boldsymbol{w}_{h}, \nabla\left(p-p_{1}\right)\right)_{K}+\nu\left(\nabla\left(\boldsymbol{u}-\boldsymbol{u}_{1}\right), \nabla \boldsymbol{w}_{h}\right)_{\Omega} \\
& +\sum_{Z \in \mathcal{E}_{h}} \tau_{Z}\left(\llbracket \nu \partial_{\boldsymbol{n}}\left(\boldsymbol{u}-\boldsymbol{u}_{1}\right) \rrbracket, \llbracket \nu \partial_{\boldsymbol{n}} \boldsymbol{w}_{h} \rrbracket\right)_{Z} \\
\leq & \sum_{K \in \mathcal{T}_{h}}\left\|\boldsymbol{w}-\boldsymbol{w}_{h}\right\|_{0, K}\left|p-p_{1}\right|_{1, K}+\nu\left|\boldsymbol{u}-\boldsymbol{u}_{1}\right|_{1, \Omega}\left|\boldsymbol{w}_{h}\right|_{1, \Omega} \\
& +\sum_{Z \in \mathcal{E}_{h}} \tau_{Z}\left\|\llbracket \nu \partial_{\boldsymbol{n}}\left(\boldsymbol{u}-\boldsymbol{u}_{1}\right) \rrbracket\right\|_{0, Z}\left\|\llbracket \nu \partial_{\boldsymbol{n}} \boldsymbol{w}_{h} \rrbracket\right\|_{0, Z} \\
\leq & {\left[\sum_{K \in \mathcal{T}_{h}} \tau_{K}\left|p-p_{1}\right|_{1, K}^{2}+\nu\left|\boldsymbol{u}-\boldsymbol{u}_{1}\right|_{1, \Omega}^{2}+\sum_{Z \in \mathcal{E}_{h}} \tau_{Z}\left\|\llbracket \nu \partial_{\boldsymbol{n}}\left(\boldsymbol{u}-\boldsymbol{u}_{1}\right) \rrbracket\right\|_{0, Z}^{2}\right]^{\frac{1}{2}} } \\
& \cdot\left[\sum_{K \in \mathcal{T}_{h}} \tau_{K}^{-1}\left\|\boldsymbol{w}-\boldsymbol{w}_{h}\right\|_{0, K}^{2}+\nu\left|\boldsymbol{w}_{h}\right|_{1, \Omega}^{2}+\sum_{Z \in \mathcal{E}_{h}} \tau_{Z}\left\|\llbracket \nu \partial_{\boldsymbol{n}} \boldsymbol{w}_{h} \rrbracket\right\|_{0, Z}^{2}\right]^{\frac{1}{2}} \\
\leq & C \sqrt{\nu}\left[\left\|\boldsymbol{u}-\boldsymbol{u}_{1}\right\|_{h}+\left\|p-p_{1}\right\|_{h}\right]\left[|\boldsymbol{w}|_{1, \Omega}^{2}+\left|\boldsymbol{w}_{h}\right|_{1, \Omega}^{2}\right]^{\frac{1}{2}} \\
\leq & C \sqrt{\nu} h\left(\sqrt{\nu}|\boldsymbol{u}|_{2, \Omega}+\frac{1}{\sqrt{\nu}}|p|_{1, \Omega}\right)\left\|p-p_{1}\right\|_{0, \Omega},
\end{aligned}
$$

where, in order to bound the term $\sum_{Z \in \mathcal{E}_{h}} \tau_{Z}\left\|\llbracket \nu \partial_{\boldsymbol{n}} \boldsymbol{w}_{h} \rrbracket\right\|_{0, Z}^{2}$ we have used the local trace result (28) and $\left.\boldsymbol{w}_{h}\right|_{K} \in \mathbb{P}^{1}(K)^{2}$. The result follows then by dividing by the last term.

4.2.2. An error estimate for $\left\|\boldsymbol{u}-\boldsymbol{u}_{1}\right\|_{0, \Omega}$. Throughout this section we will assume that the solution of the following problem, where $\left(\boldsymbol{u}_{1}, p_{1}\right)$ is the solution of (44), belongs to $\left[H^{2}(\Omega) \cap H_{0}^{1}(\Omega)\right]^{2} \times\left[H^{1}(\Omega) \cap L_{0}^{2}(\Omega)\right]$ : Find $(\boldsymbol{\varphi}, \pi)$ such that

$$
\begin{aligned}
-\nu \Delta \varphi-\nabla \pi=\boldsymbol{u}-\boldsymbol{u}_{1}, & \nabla \cdot \boldsymbol{\varphi}=0 \quad \text { in } \Omega, \\
\boldsymbol{\varphi}=\mathbf{0} \quad \text { on } \partial \Omega . &
\end{aligned}
$$

We also assume that the following estimate holds:

$$
\nu\|\boldsymbol{\varphi}\|_{2, \Omega}+\|\pi\|_{1, \Omega} \leq C\left\|\boldsymbol{u}-\boldsymbol{u}_{1}\right\|_{0, \Omega} .
$$

ThEOREM 4.11. Under the hypothesis of Theorem 4.10 the following error estimate holds:

$$
\left\|\boldsymbol{u}-\boldsymbol{u}_{1}\right\|_{0, \Omega} \leq C h^{2}\left(|\boldsymbol{u}|_{2, \Omega}+\frac{1}{\nu}|p|_{1, \Omega}\right) .
$$

Proof. Let $\left(\boldsymbol{\varphi}_{h}, \pi_{h}\right):=\left(I_{h}(\boldsymbol{\varphi}), \mathcal{C}_{h}(\pi)-\frac{\left(\mathcal{C}_{h}(\pi), 1\right)_{\Omega}}{|\Omega|}\right) \in \mathbf{V}_{h} \times Q_{h}^{1}$. Then, multiplying the first equation in (56) by $\boldsymbol{u}-\boldsymbol{u}_{1}$ and the second by $-\left(p-p_{1}\right)$, from the definition of bilinear form $\mathbf{B}$, the consistency of the method, the fact that $\llbracket \partial_{\boldsymbol{n}} \varphi \rrbracket=\mathbf{0}$ a.e. on the internal edges, interpolation inequalities (26), (27), and (35), and Theorems 4.8 
and 4.10 , we obtain

$$
\begin{aligned}
\| \boldsymbol{u} & -\boldsymbol{u}_{1} \|_{0, \Omega}^{2} \\
= & \nu\left(\nabla \boldsymbol{\varphi}, \nabla\left(\boldsymbol{u}-\boldsymbol{u}_{1}\right)\right)_{\Omega}+\left(\pi, \nabla \cdot\left(\boldsymbol{u}-\boldsymbol{u}_{1}\right)\right)_{\Omega}-\left(p-p_{1}, \nabla \cdot \boldsymbol{\varphi}\right)_{\Omega} \\
= & \mathbf{B}\left(\left(\boldsymbol{u}-\boldsymbol{u}_{1}, p-p_{1}\right),(\boldsymbol{\varphi}, \pi)\right)-\sum_{K \in \mathcal{T}_{h}} \tau_{K}\left(-\nu \Delta\left(\boldsymbol{u}-\boldsymbol{u}_{1}\right)+\nabla\left(p-p_{1}\right), \nu \Delta \boldsymbol{\varphi}+\nabla \pi\right)_{K} \\
= & \mathbf{B}\left(\left(\boldsymbol{u}-\boldsymbol{u}_{1}, p-p_{1}\right),\left(\boldsymbol{\varphi}-\boldsymbol{\varphi}_{h}, \pi-\pi_{h}\right)\right) \\
& -\sum_{K \in \mathcal{T}_{h}} \tau_{K}\left(-\nu \Delta\left(\boldsymbol{u}-\boldsymbol{u}_{1}\right)+\nabla\left(p-p_{1}\right), \nu \Delta \boldsymbol{\varphi}+\nabla \pi\right)_{K} \\
\leq & {\left[\nu\left|\boldsymbol{u}-\boldsymbol{u}_{1}\right|_{1, \Omega}^{2}+\nu\left\|\nabla \cdot\left(\boldsymbol{u}-\boldsymbol{u}_{1}\right)\right\|_{0, \Omega}^{2}+\frac{1}{\nu}\left\|p-p_{1}\right\|_{0, \Omega}^{2}\right.} \\
& \left.+\sum_{Z \in \mathcal{E}_{h}} \tau_{Z}\left\|\llbracket \nu \partial_{\boldsymbol{n}}\left(\boldsymbol{u}-\boldsymbol{u}_{1}\right) \rrbracket\right\|_{0, Z}^{2}+2 \sum_{K \in \mathcal{T}_{h}} \tau_{K}\left\|-\nu \Delta \boldsymbol{u}+\nabla\left(p-p_{1}\right)\right\|_{0, K}^{2}\right]^{\frac{1}{2}} \cdot \\
& {\left[\nu\left|\boldsymbol{\varphi}-\boldsymbol{\varphi}_{h}\right|_{1, \Omega}^{2}+\frac{1}{\nu}\left\|\pi-\pi_{h}\right\|_{0, \Omega}^{2}+\nu\left\|\nabla \cdot\left(\boldsymbol{\varphi}-\boldsymbol{\varphi}_{h}\right)\right\|_{0, \Omega}^{2}+\sum_{K \in \mathcal{T}_{h}} \tau_{K}\left\|\nabla\left(\pi-\pi_{h}\right)\right\|_{0, K}^{2}\right.} \\
& \left.+\sum_{Z \in \mathcal{E}_{h}} \tau_{Z}\left\|\llbracket \nu \partial_{\boldsymbol{n}}\left(\boldsymbol{\varphi}-\boldsymbol{\varphi}_{h}\right) \rrbracket\right\|_{0, Z}^{2}+\sum_{K \in \mathcal{T}_{h}} \tau_{K}\|\nu \Delta \boldsymbol{\varphi}+\nabla \pi\|_{0, K}^{2}\right]^{\frac{1}{2}} \\
\leq & C\left[\left\|\boldsymbol{u}-\boldsymbol{u}_{1}\right\|_{h}^{2}+\nu h^{2}|\boldsymbol{u}|_{2, \Omega}^{2}+\left\|p-p_{1}\right\|_{h}^{2}+\frac{1}{\nu}\left\|p-p_{1}\right\|_{0, \Omega}^{2}\right]^{\frac{1}{2}}\left[\nu h^{2}|\boldsymbol{\varphi}|_{2, \Omega}^{2}+\frac{h^{2}}{\nu}|\pi|_{1, \Omega}^{2}\right]^{\frac{1}{2}} \\
\leq & C \frac{1}{\sqrt{\nu}} h^{2}\left(\sqrt{\nu}|\boldsymbol{u}|_{2, \Omega}+\frac{1}{\sqrt{\nu}}|p|_{1, \Omega}\right)\left\|\boldsymbol{u}-\boldsymbol{u}_{1}\right\|_{0, \Omega},
\end{aligned}
$$

and the result follows.

REMARK 4.12. As we claimed before, the error analysis is independent of the nature of the $\boldsymbol{f}$ on the right-hand side, and hence, we have actually justified method (44) for a general $\boldsymbol{f} \in L^{2}(\Omega)^{2}$. In Appendix B we will show that if $\boldsymbol{f} \in H^{1}(\Omega)^{2}$, then the difference between implementing method (44) and $\left(\boldsymbol{f}, \boldsymbol{v}_{1}\right)_{\Omega}+\sum_{K \in \mathcal{T}_{h}} \nu^{-1}\left(\mathcal{M}_{K}(\boldsymbol{f}), \nu \Delta \boldsymbol{v}_{1}+\right.$ $\nabla q)_{K}$ on the right-hand side is smaller than the order of the method. On the other hand, method (44) has been justified for any constant $C_{1}>0$, even if it has been presented with $C_{1}=\frac{1}{8}$.

5. An alternative formulation including the residual on the boundary. In this section we propose another class of methods arising from a different choice of enrichment functions. We will denote by $R_{h}$ the pressure space according to the choice of elements, i.e., $R_{h}=Q_{h}^{1}$ for $\mathbb{P}^{1} / \mathbb{P}^{1}$ elements and $R_{h}=Q_{h}^{0}$ for $\mathbb{P}^{1} / \mathbb{P}^{0}$ elements. The proposed method reads as follows: Find $\left(\boldsymbol{u}^{r}, p^{r}\right) \in \mathbf{V}_{h} \times R_{h}$ such that

$$
\mathbf{B}_{r}\left(\left(\boldsymbol{u}^{r}, p^{r}\right),\left(\boldsymbol{v}_{1}, q\right)\right)=\mathbf{F}\left(\boldsymbol{v}_{1}, q\right) \quad \forall\left(\boldsymbol{v}_{1}, q\right) \in \mathbf{V}_{h} \times R_{h},
$$

where

$$
\begin{aligned}
& \mathbf{B}_{r}\left(\left(\boldsymbol{u}_{1}, p\right),\left(\boldsymbol{v}_{1}, q\right)\right)=\nu\left(\nabla \boldsymbol{u}_{1}, \nabla \boldsymbol{v}_{1}\right)_{\Omega}-\left(p, \nabla \cdot \boldsymbol{v}_{1}\right)_{\Omega}+\left(q, \nabla \cdot \boldsymbol{u}_{1}\right)_{\Omega} \\
& \quad+\sum_{K \in \mathcal{T}_{h}} \tau_{K}\left(-\nu \Delta \boldsymbol{u}_{1}+\nabla p, \nu \Delta \boldsymbol{v}_{1}+\nabla q\right)_{K} \\
& \quad+\sum_{Z \in \mathcal{E}_{h}} \tilde{\tau}_{Z}\left(\llbracket-\nu \partial_{\boldsymbol{n}} \boldsymbol{u}_{1}+p \mathbf{I} \cdot \boldsymbol{n} \rrbracket, \llbracket \nu \partial_{\boldsymbol{n}} \boldsymbol{v}_{1}+q \mathbf{I} \cdot \boldsymbol{n} \rrbracket\right)_{Z},
\end{aligned}
$$


$\mathbf{F}$ is given by (42), $\tau_{K}$ by (43), and

$$
\tilde{\tau}_{Z}:=\frac{h_{Z}}{12 \alpha \nu}
$$

where $\alpha>0$ will be fixed in order to have a well-posed problem.

This method may be obtained in the same way as method (14) and (44) by taking the enrichment function $\boldsymbol{u}_{e}$ to be the solution of (4), together with the boundary conditions

$$
-\nu \partial_{\boldsymbol{s s}} \boldsymbol{u}_{e}=\frac{1}{\alpha h_{Z}} \llbracket-\nu \partial_{\boldsymbol{n}} \boldsymbol{u}_{1}^{r}+p^{r} \mathbf{I} \cdot \boldsymbol{n} \rrbracket \text { on each } Z \subset \partial K, \quad \boldsymbol{u}_{e}=\mathbf{0} \text { at the nodes },
$$

on the internal edges, and $\boldsymbol{u}_{e}=\mathbf{0}$ on $\partial K \cap \partial \Omega$. In fact, using this choice of enrichment we can perform the same derivation from sections 3 and 4 , neglecting once more a cross term appearing in $\mathbb{P}^{1} / \mathbb{P}^{1}$ discretization.

REMARK 5.1. This method is different from (14) and (44) from two viewpoints. First, the boundary term contains the residual of the Cauchy stress tensor on the trial function. This fact comes from the choice of the enriched part as being a corrector for the residual inside the element and on the boundary. The other difference is the stabilization parameter on the edges. Now, this parameter contains a constant to set.

Now, let $\|\cdot\|_{h}$ be the mesh-dependent norm defined by:

$$
\left\|\left(\boldsymbol{v}_{1}, q\right)\right\|_{h}:=\left[\nu\left|\boldsymbol{v}_{1}\right|_{1, \Omega}^{2}+\sum_{K \in \mathcal{T}_{h}} \tau_{K}|q|_{1, K}^{2}+\sum_{Z \in \mathcal{E}_{h}} \tilde{\tau}_{Z}\|\llbracket q \rrbracket\|_{0, Z}^{2}\right]^{\frac{1}{2}} .
$$

Then, we have the following coercivity result.

Lemma 5.2. Let us suppose that $\alpha>C_{t} / 3$, where $C_{t}>0$ is the constant from local trace result (28). Then, for all $\left(\boldsymbol{v}_{1}, q\right) \in \mathbf{V}_{h} \times R_{h}$ there holds

$$
\mathbf{B}_{r}\left(\left(\boldsymbol{v}_{1}, q\right),\left(\boldsymbol{v}_{1}, q\right)\right) \geq \frac{1}{2}\left\|\left(\boldsymbol{v}_{1}, q\right)\right\|_{h}^{2} .
$$

Proof. Let $\left(\boldsymbol{v}_{1}, q\right) \in \mathbf{V}_{h} \times R_{h}$. Then, since $\Delta \boldsymbol{v}_{1}=\mathbf{0}$ on each $K \in \mathcal{T}_{h}$, applying local trace result (28) and the definition of $\tilde{\tau}_{Z}$ we obtain

$$
\begin{aligned}
\mathbf{B}_{r} & \left(\left(\boldsymbol{v}_{1}, q\right),\left(\boldsymbol{v}_{1}, q\right)\right)=\nu\left|\boldsymbol{v}_{1}\right|_{1, \Omega}^{2} \\
& +\sum_{K \in \mathcal{T}_{h}} \tau_{K}\|\nabla q\|_{0, K}^{2}+\sum_{Z \in \mathcal{E}_{h}} \tilde{\tau}_{Z}\left(-\nu^{2}\left\|\llbracket \partial_{\boldsymbol{n}} \boldsymbol{v}_{1} \rrbracket\right\|_{0, Z}^{2}+\|\llbracket q \rrbracket\|_{0, Z}^{2}\right) \\
\geq & \nu\left|\boldsymbol{v}_{1}\right|_{1, \Omega}^{2}-\frac{C_{t}}{6 \alpha} \nu \sum_{K \in \mathcal{T}_{h}}\left|\boldsymbol{v}_{1}\right|_{1, K}^{2}+\sum_{K \in \mathcal{T}_{h}} \tau_{K}|q|_{1, K}^{2}+\sum_{Z \in \mathcal{E}_{h}} \tilde{\tau}_{Z}\|\llbracket q \rrbracket\|_{0, Z}^{2} \\
\geq & \frac{1}{2}\left\|\left(\boldsymbol{v}_{1}, q\right)\right\|_{h}^{2},
\end{aligned}
$$

an the result follows.

Once this method has been proved to be stable, following a procedure absolutely analogous to those from sections 3 and 4 we can prove the consistency of (58) and perform a complete error analysis of (58), obtaining the same results as in previous sections. 


\section{Numerical validations.}

6.1. An analytical solution: Convergence validation. For this test case, the domain is taken as the square $\Omega=(0,1) \times(0,1), \nu=1$, and $\boldsymbol{f}$ is set such that the exact solution of our Stokes problem is given by

$$
\begin{aligned}
u_{1}(x, y) & =-256 x^{2}(x-1)^{2} y(y-1)(2 y-1), \\
u_{2}(x, y) & =-u_{1}(y, x), \\
p(x, y) & =150(x-0.5)(y-0.5) .
\end{aligned}
$$

We perform convergence analysis for methods (14), (44), and (58) using continuous $\mathbb{P}^{1} / \mathbb{P}^{1}$ and $\mathbb{P}^{1} / \mathbb{P}^{0}$ elements.

6.1.1. The $\mathbb{P}^{\mathbf{1}} / \mathbb{P}^{\mathbf{1}}$ case. For this case we first depict in Figures $1-2$ the convergence history for method (44). The results reproduce our theoretical results showing an $O(h)$ order of convergence for $\left|\boldsymbol{u}-\boldsymbol{u}_{1}\right|_{1, \Omega}$,

$$
\left|\llbracket \partial_{\boldsymbol{n}}\left(\boldsymbol{u}-\boldsymbol{u}_{1}\right) \rrbracket\right|_{h}:=\left[\sum_{Z \in \mathcal{E}_{h}} h_{Z}\left\|\llbracket \partial_{\boldsymbol{n}}\left(\boldsymbol{u}-\boldsymbol{u}_{1}\right) \rrbracket\right\|_{0, Z}^{2}\right]^{\frac{1}{2}}
$$

and $\left\|p-p_{1}\right\|_{0, \Omega}$, and an $O\left(h^{2}\right)$ convergence for $\left\|\boldsymbol{u}-\boldsymbol{u}_{1}\right\|_{0, \Omega}$.
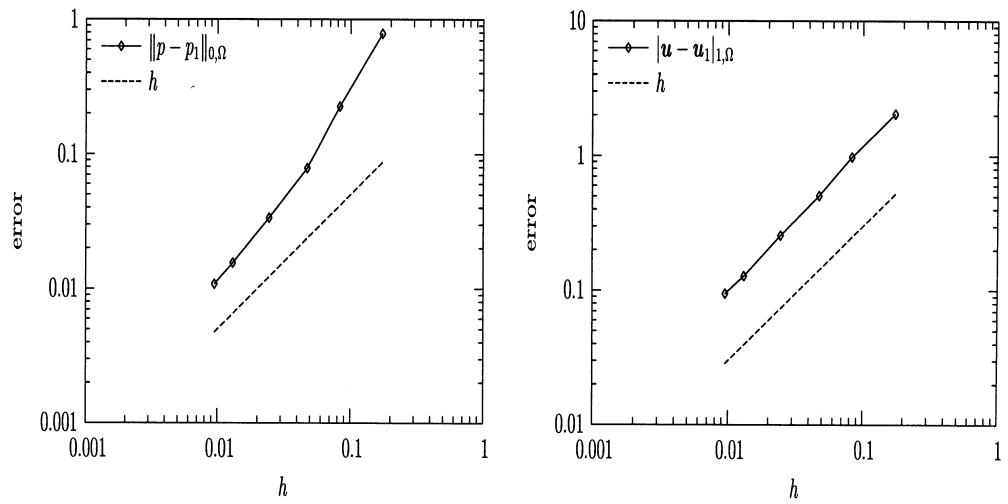

FIG. 1. Method (44): convergence history for $\left\|p-p_{1}\right\|_{0, \Omega}$ and $\left|\boldsymbol{u}-\boldsymbol{u}_{1}\right|_{1, \Omega}$.
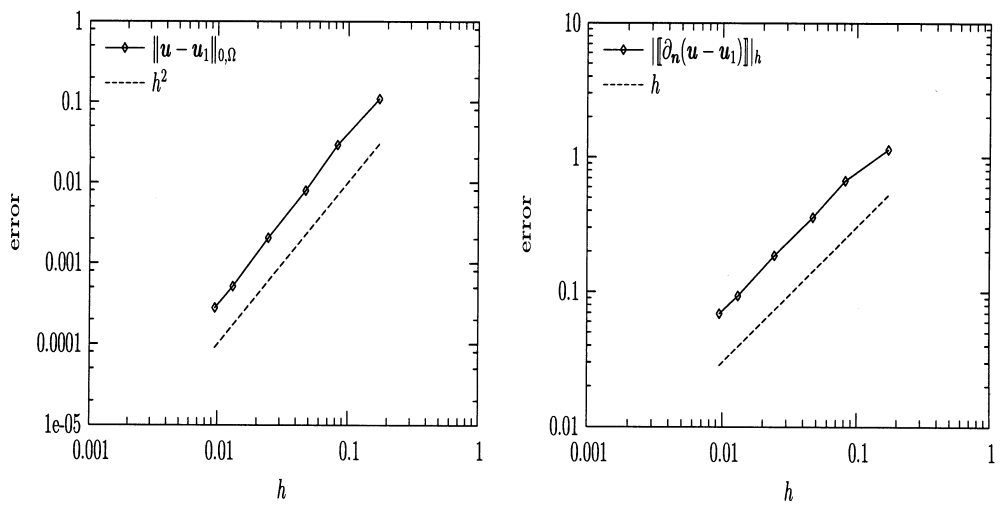

FIG. 2. Method (44): convergence history for $\left\|\boldsymbol{u}-\boldsymbol{u}_{1}\right\|_{0, \Omega}$ and $\left|\llbracket \partial_{\boldsymbol{n}}\left(\boldsymbol{u}-\boldsymbol{u}_{1}\right) \rrbracket\right|_{h}$. 

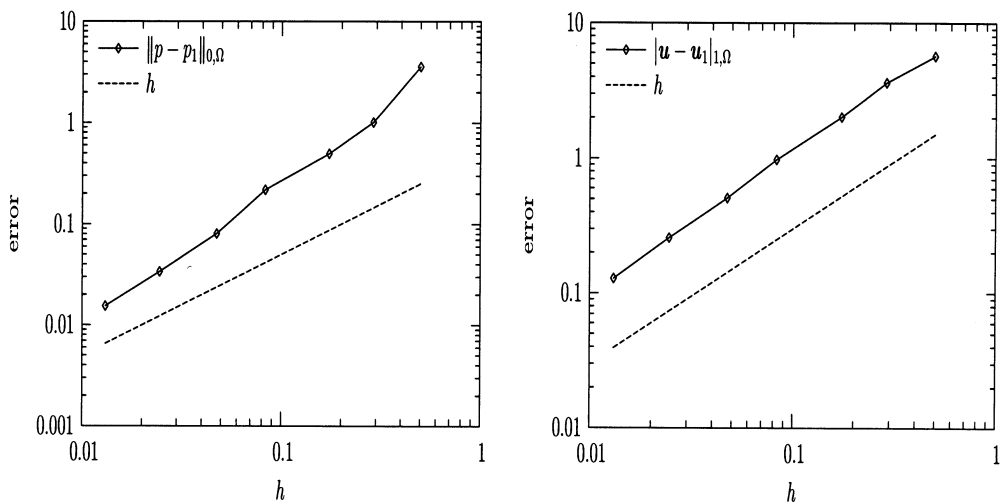

FIG. 3. Method (58): convergence history for $\left\|p-p_{1}\right\|_{0, \Omega}$ and $\left|\boldsymbol{u}-\boldsymbol{u}_{1}\right|_{1, \Omega}$.
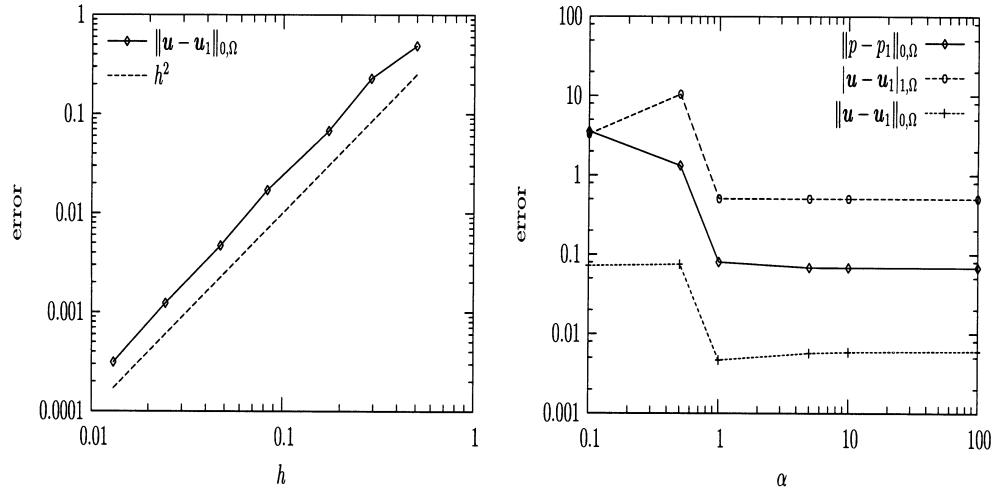

FIG. 4. Method (58): convergence history for $\left\|\boldsymbol{u}-\boldsymbol{u}_{1}\right\|_{0, \Omega}$, and sensitivity of (58) with respect to $\alpha$.

Method (58) is tested next. The results are depicted in Figures $3-4$ using $\alpha=1$, where the results are in perfect accordance with the theoretical results. The justification of this choice for $\alpha$ may be found in Figure 4 (on the right) where we have depicted the behavior of the error in terms of $\alpha$ (using a mesh of around 2500 elements) and we see that for $\alpha \geq 1$ the error is almost independent of $\alpha$, showing that the restriction of Lemma 5.2 is not only theoretical, but at the same time showing that, once we are inside the region predicted by the theory, the performance of the method is independent of $\alpha$.

6.1.2. The $\mathbb{P}^{\mathbf{1}} / \mathbb{P}^{\mathbf{0}}$ case. For this case we first depict in Figures $5-6$ the convergence history for method (14). The results reproduce our theoretical results showing an $O(h)$ order of convergence for $\left|\boldsymbol{u}-\boldsymbol{u}_{1}\right|_{1, \Omega},\left|\llbracket \partial_{\boldsymbol{n}}\left(\boldsymbol{u}-\boldsymbol{u}_{1}\right)+\left(p-p_{0}\right) \boldsymbol{n} \rrbracket\right|_{h}$ and $\left\|p-p_{0}\right\|_{0, \Omega}$, and an $O\left(h^{2}\right)$ convergence for $\left\|\boldsymbol{u}-\boldsymbol{u}_{1}\right\|_{0, \Omega}$.

Method (58) is tested next. The results are depicted in Figures 7-8 using $\alpha=1$, where the results are in perfect accordance with the theoretical results, giving an $O(h)$ for $\left|\boldsymbol{u}-\boldsymbol{u}_{1}\right|_{1, \Omega},\left|\llbracket p-p_{0} \rrbracket\right|_{h}$, and $\left\|p-p_{0}\right\|_{0, \Omega}$, and an $O\left(h^{2}\right)$ convergence for $\left\|\boldsymbol{u}-\boldsymbol{u}_{1}\right\|_{0, \Omega}$. Concerning the choice of $\alpha$, the situation now is quite different from that in the previous section. As a matter of fact, since we only control the pressure 

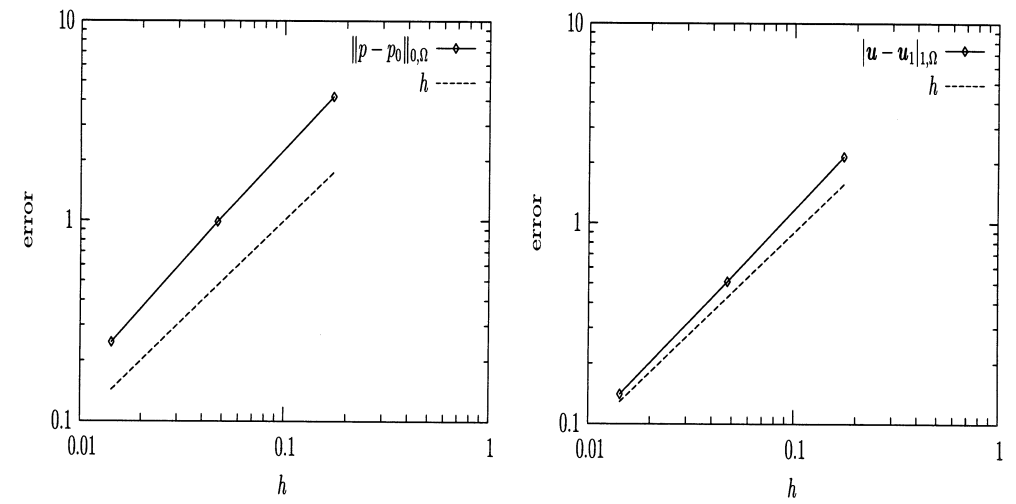

FIG. 5. Method (14): convergence history for $\left\|p-p_{0}\right\|_{0, \Omega}$ and $\left|\boldsymbol{u}-\boldsymbol{u}_{1}\right|_{1, \Omega}$.
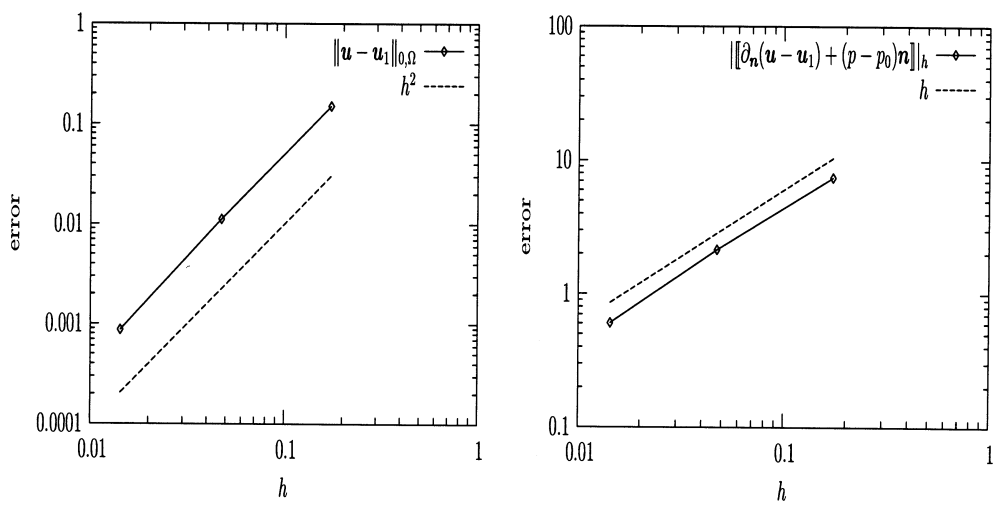

FIG. 6. Method (14): convergence history for $\left\|\boldsymbol{u}-\boldsymbol{u}_{1}\right\|_{0, \Omega}$ and $\left|\llbracket \partial_{\boldsymbol{n}}\left(\boldsymbol{u}-\boldsymbol{u}_{1}\right)+\left(p-p_{0}\right) \boldsymbol{n} \rrbracket\right|_{h}$.

via the jump terms governed by $\alpha$, we can expect the error to grow as $\alpha$ grows, as it is shown in Figure 9 (for a mesh of 2500 elements) where we see that all the errors attain a minimum at $\alpha=1$ (i.e., using $\tilde{\tau}_{Z}=\tau_{Z}$ ), and then they present a growing behavior. Values larger than 10 have been tested and the behavior is growing in all the errors. Related experiments have been performed using the GLS method (cf. [27]), obtaining similar results.

6.2. The lid-driven cavity problem. For this case we use the same domain as in the previous section, we set $\boldsymbol{f}=\mathbf{0}$, and the boundary conditions $\boldsymbol{u}=\mathbf{0}$ on $[\{0\} \times(0,1)] \cup[(0,1) \times\{0\}] \cup[\{1\} \times(0,1)]$ and $\boldsymbol{u}=(1,0)^{t}$ on $(0,1) \times\{1\}$. In Figure 10 we depict the pressure isovalues for both $\mathbb{P}^{1} / \mathbb{P}^{0}$ and $\mathbb{P}^{1} / \mathbb{P}^{1}$ approximations (using a mesh of around 1000 elements) showing, in both cases, the absence of oscillations.

7. Concluding remarks. In this paper we have analyzed and tested new stabilized finite element methods for the Stokes problem. These new methods arise from multiscale enrichment of the trial space for the velocity coupled with a PetrovGalerkin strategy. This Petrov-Galerkin strategy makes it possible to perform statical condensation both at the element level and at the interelement boundary level, making the method take the form of a classical stabilized finite element method, containing jump terms on the interior edges of the triangulation, and with the corresponding 

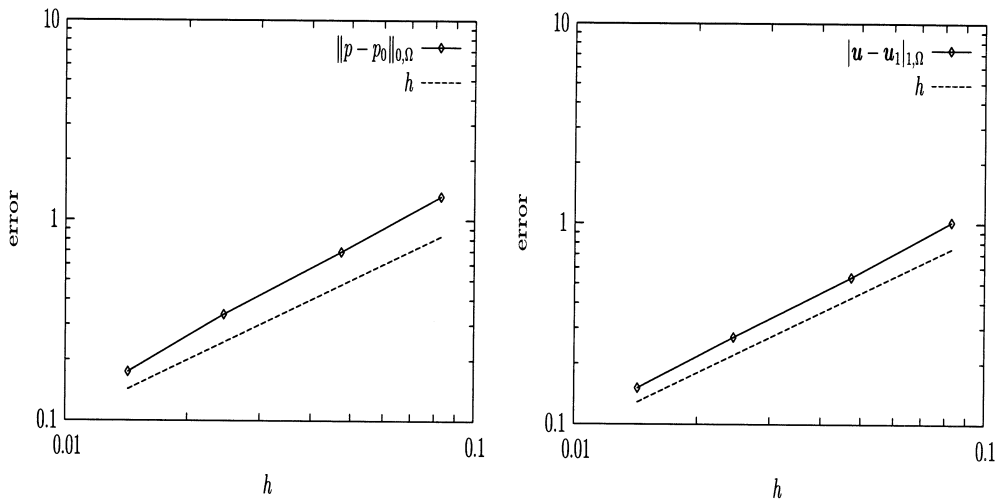

FIG. 7. Method (58): convergence history for $\left\|p-p_{0}\right\|_{0, \Omega}$ and $\left|\boldsymbol{u}-\boldsymbol{u}_{1}\right|_{1, \Omega}$.
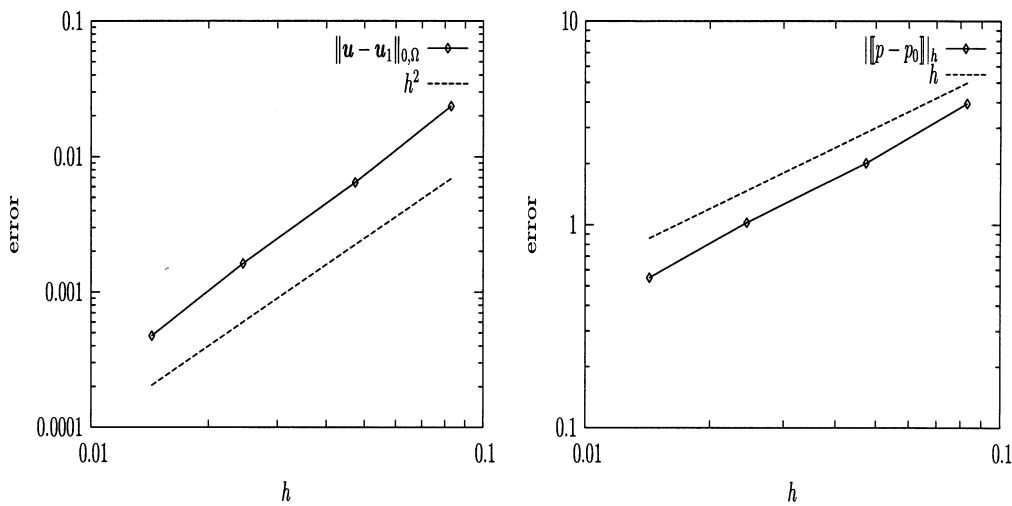

FIG. 8. Method (58): convergence history for $\left\|\boldsymbol{u}-\boldsymbol{u}_{1}\right\|_{0, \Omega}$ and $\left\|p-p_{0}\right\|_{0, \Omega}$.

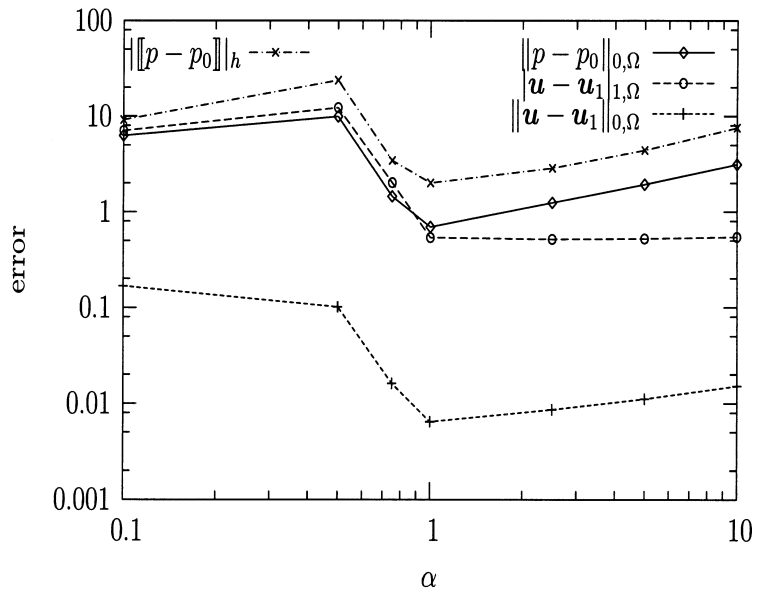

FiG. 9. Sensitivity of method (58) to $\alpha$. 

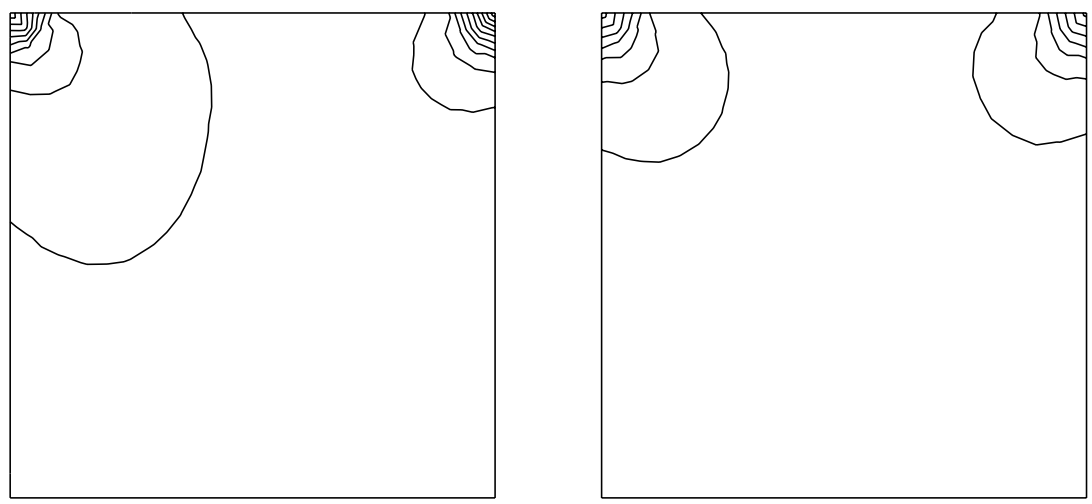

FIG. 10. Pressure isovalues for $\mathbb{P}^{1} / \mathbb{P}^{0}$ (left) and $\mathbb{P}^{1} / \mathbb{P}^{1}$ (right) approximations.

stabilization parameter known exactly. Optimal order error estimates were derived using the natural norms, results that were confirmed by the numerical experiments.

Our belief is that our general methodology may be applied to other mixed problems, namely the Darcy and Brinkman flow problems, and to the advection-diffusion equation. This will be the subject of future works.

\section{Appendix A. Proof of Theorem 4.3.}

The consistency of the method is immediate from the fact that $\llbracket \nu \partial_{\boldsymbol{n}} \boldsymbol{u} \rrbracket=\mathbf{0}$ a.e. on $\partial K$. To prove the well-posedness of (39), we prove that $\mathbf{B}_{1}$ is an elliptic bilinear form. Let $\left(\boldsymbol{v}_{1}, q_{1}\right) \in \mathbf{V}_{h} \times Q_{h}^{1}$; then from Lemma 4.6 we have that

$$
\mathbf{B}_{1}\left(\left(\boldsymbol{v}_{1}, q_{1}\right),\left(\boldsymbol{v}_{1}, q_{1}\right)\right)=\left\|\boldsymbol{v}_{1}\right\|_{h}^{2}+\left\|q_{1}\right\|_{h}^{2}-\sum_{K \in \mathcal{T}_{h}} \frac{1}{\nu}\left(\mathcal{B}_{K}\left(\llbracket \nu \partial_{\boldsymbol{n}} \boldsymbol{v}_{1} \rrbracket\right), \nabla q_{1}\right)_{K}
$$

Now, in order to treat the last term above, let us denote by $Z_{1}, Z_{2}, Z_{3}$ the sides of $K$, and let, for $i=1,2,3, b_{K}^{Z_{i}}$ be the solution of

$$
-\Delta b_{K}^{Z_{i}}=0 \quad \text { in } K, \quad b_{K}^{Z_{i}}=g_{i} \quad \text { on each } Z \subset \partial K
$$

where $g_{i}=0$ if $Z_{i} \subseteq \partial \Omega$, and $g_{i}$ is the solution of

$$
-\partial_{s s} g_{i}=\frac{1}{h_{Z_{i}}} \quad \text { in } Z_{i}, \quad g_{i}=0 \quad \text { on } \partial K-Z_{i},
$$

otherwise. First, we remark that from the maximum principle, we have that $0 \leq b_{K}^{Z_{i}} \leq$ $\frac{h_{Z_{i}}}{8}$ in $K$. On the other hand, it is easy to see that $\mathcal{B}_{K}\left(\llbracket \nu \partial_{\boldsymbol{n}} \boldsymbol{v}_{1} \rrbracket\right)=\left.\sum_{i=1}^{3} b_{K}^{Z_{i}} \llbracket \nu \partial_{\boldsymbol{n}} \boldsymbol{v}_{1} \rrbracket\right|_{Z_{i}}$, and then, using that $|K| \leq \frac{h_{K}^{2}}{2}$ and the inequality $a b \leq \gamma^{-1} \frac{a^{2}}{4}+\gamma b^{2}(\gamma>0)$ (denoting 
$\|\cdot\|_{\mathbb{R}^{2}}$ the Euclidean norm on $\mathbb{R}^{2}$ ) we arrive at

$$
\begin{aligned}
& \sum_{K \in \mathcal{T}_{h}} \frac{1}{\nu}\left(\mathcal{B}_{K}\left(\llbracket \nu \partial_{\boldsymbol{n}} \boldsymbol{v}_{1} \rrbracket\right), \nabla q_{1}\right)_{K}=\sum_{K \in \mathcal{T}_{h}} \sum_{i=1}^{3} \frac{1}{\nu}\left(\left.b_{K}^{Z} \llbracket \nu \partial_{\boldsymbol{n}} \boldsymbol{v}_{1} \rrbracket\right|_{Z_{i}}, \nabla q_{1}\right)_{K} \\
& =\left.\left.\sum_{Z \in \mathcal{E}_{h}} \sum_{K \subset \omega_{Z}} \frac{\left(b_{K}^{Z}, 1\right)_{K}}{\nu} \llbracket \nu \partial_{\boldsymbol{n}} \boldsymbol{v}_{1} \rrbracket\right|_{Z} \cdot \nabla q_{1}\right|_{K} \\
& \leq \sum_{Z \in \mathcal{E}_{h}} \sum_{K \subset \omega_{Z}} \frac{h_{Z}|K|}{8 \nu}\left\|\left.\llbracket \nu \partial_{\boldsymbol{n}} \boldsymbol{v}_{1} \rrbracket\right|_{Z}\right\|_{\mathbb{R}^{2}}\left\|\left.\nabla q_{1}\right|_{K}\right\|_{\mathbb{R}^{2}} \\
& \leq \gamma^{-1} \sum_{Z \in \mathcal{E}_{h}} \sum_{K \subset \omega_{Z}} \frac{|Z|\left\|\llbracket \nu \partial_{\boldsymbol{n}} \boldsymbol{v}_{1} \rrbracket\right\|_{0, Z}^{2}}{32 \nu}+\gamma \sum_{Z \in \mathcal{E}_{h}} \sum_{K \subset \omega_{Z}} \frac{|K|\left\|\nabla q_{1}\right\|_{0, K}^{2}}{8 \nu} \\
& \leq \gamma^{-1} \sum_{Z \in \mathcal{E}_{h}} \frac{h_{Z}\left\|\llbracket \partial_{\boldsymbol{n}} \boldsymbol{v}_{1} \rrbracket\right\|_{0, Z}^{2}}{16 \nu}+\gamma \sum_{K \in \mathcal{T}_{h}} \frac{h_{K}^{2}\left\|\nabla q_{1}\right\|_{0, K}^{2}}{8 \nu}
\end{aligned}
$$

Hence, choosing $\gamma=\frac{14}{16}<1$ we arrive at

$$
\begin{aligned}
\mathbf{B}_{1}\left(\left(\boldsymbol{v}_{1}, q_{1}\right),\left(\boldsymbol{v}_{1}, q_{1}\right)\right) & \geq\left\|\boldsymbol{v}_{1}\right\|_{h}^{2}+\left\|q_{1}\right\|_{h}^{2}-\sum_{Z \in \mathcal{E}_{h}} \frac{h_{Z}}{14 \nu}\left\|\llbracket \nu \partial_{\boldsymbol{n}} \boldsymbol{v}_{1} \rrbracket\right\|_{0, Z}^{2}-\gamma \sum_{K \in \mathcal{T}_{h}} \frac{h_{K}^{2}}{8 \nu}\left|q_{1}\right|_{1, K}^{2} \\
& \geq C_{*}\left(\left\|\boldsymbol{v}_{1}\right\|_{h}^{2}+\left\|q_{1}\right\|_{h}^{2}\right),
\end{aligned}
$$

where $C_{*}$ is a positive constant not depending on $h$ or $\nu$. Now, for the error estimate, applying the coercivity result and the consistency of the method we arrive at

$$
\begin{aligned}
C_{*}\left(\left\|\boldsymbol{u}_{1}-\tilde{\boldsymbol{u}}_{1}\right\|_{h}^{2}+\left\|p_{1}-\tilde{p}_{1}\right\|_{h}^{2}\right) & \leq \mathbf{B}_{1}\left(\left(\boldsymbol{u}_{1}-\tilde{\boldsymbol{u}}_{1}, p_{1}-\tilde{p}_{1}\right),\left(\boldsymbol{u}_{1}-\tilde{\boldsymbol{u}}_{1}, p_{1}-\tilde{p}_{1}\right)\right) \\
& =\mathbf{B}_{1}\left(\left(\boldsymbol{u}_{1}-\boldsymbol{u}, p_{1}-p\right),\left(\boldsymbol{u}_{1}-\tilde{\boldsymbol{u}}_{1}, p_{1}-\tilde{p}_{1}\right)\right) \\
& =-\sum_{K \in \mathcal{T}_{h}}\left(\mathcal{B}_{K}\left(\llbracket \nu \partial_{\boldsymbol{n}}\left(\boldsymbol{u}_{1}-\boldsymbol{u}\right) \rrbracket\right), \nabla\left(p_{1}-\tilde{p}_{1}\right)\right)_{K} .
\end{aligned}
$$

Finally, proceeding as in (63) it is not difficult to see that

$$
\begin{aligned}
\sum_{K \in \mathcal{T}_{h}} & \left(\mathcal{B}_{K}\left(\llbracket \nu \partial_{\boldsymbol{n}}\left(\boldsymbol{u}_{1}-\boldsymbol{u}\right) \rrbracket\right), \nabla\left(p_{1}-\tilde{p}_{1}\right)\right)_{K} \\
& \leq C \sum_{Z \in \mathcal{E}_{h}} \tau_{Z}\left\|\llbracket \nu \partial_{\boldsymbol{n}}\left(\boldsymbol{u}_{1}-\boldsymbol{u}\right) \rrbracket\right\|_{0, Z}^{2}+\frac{C_{*}}{2} \sum_{K \in \mathcal{T}_{h}} \tau_{K}\left|p_{1}-\tilde{p}_{1}\right|_{1, K}^{2} \\
& \leq C\left(\left\|\boldsymbol{u}-\boldsymbol{u}_{1}\right\|_{h}^{2}+\left\|p-p_{1}\right\|_{h}^{2}\right)+\frac{C_{*}}{2}\left(\left\|\boldsymbol{u}_{1}-\tilde{\boldsymbol{u}}_{1}\right\|_{h}^{2}+\left\|p_{1}-\tilde{p}_{1}\right\|_{h}^{2}\right),
\end{aligned}
$$

and hence, there exists $C>0$, independent of $h$ and $\nu$, such that

$$
\left\|\boldsymbol{u}_{1}-\tilde{\boldsymbol{u}}_{1}\right\|_{h}^{2}+\left\|p_{1}-\tilde{p}_{1}\right\|_{h}^{2} \leq C\left(\left\|\boldsymbol{u}-\boldsymbol{u}_{1}\right\|_{h}^{2}+\left\|p-p_{1}\right\|_{h}^{2}\right),
$$

and the result follows by triangular inequality.

REMARK A.1. We have proved that the error of method (39) is bounded by the error of method (44). The same analysis of Theorems 4.10 and 4.11 may be carried out to prove error estimates on $\left\|p-\tilde{p}_{1}\right\|_{0, \Omega}$ and $\left\|\boldsymbol{u}-\tilde{\boldsymbol{u}}_{1}\right\|_{0, \Omega}$.

Appendix B. The error if $f$ is not piecewise constant. As we claimed before, we have assumed that $\boldsymbol{f}$ is piecewise constant in order to derive (44), but this 
assumption does not affect the convergence of the method, and hence (44) may be implemented as it is presented for a general function $\boldsymbol{f} \in L^{2}(\Omega)^{2}$. Now, if we do not suppose that $\boldsymbol{f}$ is piecewise constant in the derivation, then method (44) becomes the following: Find $\left(\boldsymbol{u}_{h}, p_{h}\right) \in \mathbf{V}_{h} \times Q_{h}^{1}$ such that

$$
\mathbf{B}\left(\left(\boldsymbol{u}_{h}, p_{h}\right),\left(\boldsymbol{v}_{1}, q_{1}\right)\right)=\mathbf{F}_{h}\left(\boldsymbol{v}_{1}, q_{1}\right)
$$

for all $\left(\boldsymbol{v}_{1}, q_{1}\right) \in \mathbf{V}_{h} \times Q_{h}^{1}$, where $\mathbf{B}$ is defined in (41) and $\mathbf{F}_{h}$ is given by

$$
\mathbf{F}_{h}\left(\boldsymbol{v}_{1}, q_{1}\right):=\left(\boldsymbol{f}, \boldsymbol{v}_{1}\right)_{\Omega}+\sum_{K \in \mathcal{T}_{h}} \frac{1}{\nu}\left(\mathcal{M}_{K}(\boldsymbol{f}), \nabla q_{1}\right)_{K} .
$$

Clearly, (65) has a unique solution $\left(\boldsymbol{u}_{h}, p_{h}\right) \in \mathbf{V}_{h} \times Q_{h}^{1}$. Moreover, the following result holds.

THEOREM B.1. Let us suppose that $\boldsymbol{f} \in H^{1}(\Omega)^{2}$. Then, under the hypothesis of Theorems 4.8, 4.10, and 4.11, the following error estimate holds:

$$
\begin{aligned}
\left\|\boldsymbol{u}-\boldsymbol{u}_{h}\right\|_{h}+\left\|p-p_{h}\right\|_{h} & \leq C h\left(\sqrt{\nu}|\boldsymbol{u}|_{2, \Omega}+\frac{1}{\sqrt{\nu}}|p|_{1, \Omega}+\frac{1}{\sqrt{\nu}}\|\boldsymbol{f}\|_{1, \Omega}\right), \\
\left\|p-p_{h}\right\|_{0, \Omega} & \leq C h\left(\nu|\boldsymbol{u}|_{2, \Omega}+|p|_{1, \Omega}+\|\boldsymbol{f}\|_{1, \Omega}\right), \\
\left\|\boldsymbol{u}-\boldsymbol{u}_{h}\right\|_{0, \Omega} & \leq C h^{2}\left(|\boldsymbol{u}|_{2, \Omega}+\frac{1}{\nu}|p|_{1, \Omega}+\frac{1}{\nu}\|\boldsymbol{f}\|_{1, \Omega}\right) .
\end{aligned}
$$

Proof. Let $\left(\boldsymbol{u}_{1}, p_{1}\right)$ be the solution of (44). First, applying [30, Lem. 5.3.1], we see that

$$
\begin{aligned}
\left\|\boldsymbol{u}_{1}-\boldsymbol{u}_{h}\right\|_{h}+\left\|p_{1}-p_{h}\right\|_{h} & \leq \sup _{\left(\boldsymbol{v}_{1}, q_{1}\right) \in \mathbf{V}_{h} \times Q_{h}^{1}-\{\theta\}} \frac{\mathbf{F}\left(\boldsymbol{v}_{1}, q_{1}\right)-\mathbf{F}_{h}\left(\boldsymbol{v}_{1}, q_{1}\right)}{\left\|\boldsymbol{v}_{1}\right\|_{h}+\left\|q_{1}\right\|_{h}} \\
& =\sup _{\left(\boldsymbol{v}_{1}, q_{1}\right) \in \mathbf{V}_{h} \times Q_{h}^{1}-\{\theta\}} \frac{\sum_{K \in \mathcal{T}_{h}}\left(\tau_{K} \boldsymbol{f}-\frac{1}{\nu} \mathcal{M}_{K}(\boldsymbol{f}), \nabla q_{1}\right)_{K}}{\left\|\boldsymbol{v}_{1}\right\|_{h}+\left\|q_{1}\right\|_{h}} \\
& \leq \sup _{\left(\boldsymbol{v}_{1}, q_{1}\right) \in \mathbf{V}_{h} \times Q_{h}^{1}-\{\theta\}} \frac{\sum_{K \in \mathcal{T}_{h}}\left\|\tau_{K} \boldsymbol{f}-\frac{1}{\nu} \mathcal{M}_{K}(\boldsymbol{f})\right\|_{0, K}\left|q_{1}\right|_{1, K}}{\left\|\boldsymbol{v}_{1}\right\|_{h}+\left\|q_{1}\right\|_{h}} .
\end{aligned}
$$

Now, let $\boldsymbol{f}_{h}$ be the piecewise constant function given by

$$
\left.\boldsymbol{f}_{h}\right|_{K}=\frac{1}{|K|} \int_{K} \boldsymbol{f}
$$

This function, which is the (local) projection on the space of piecewise constant functions, satisfies (cf. [17]) $\left\|\boldsymbol{f}-\boldsymbol{f}_{h}\right\|_{0, K} \leq C h_{K}|\boldsymbol{f}|_{1, K}$. Then, applying triangular inequality we arrive at

$$
\begin{aligned}
\left\|\tau_{K} \boldsymbol{f}-\mathcal{M}_{K}(\boldsymbol{f})\right\|_{0, K} \leq & \left\|\tau_{K}\left(\boldsymbol{f}-\boldsymbol{f}_{h}\right)\right\|_{0, K}+\left\|\tau_{K} \boldsymbol{f}_{h}-\frac{1}{\nu} \mathcal{M}_{K}\left(\boldsymbol{f}_{h}\right)\right\|_{0, K} \\
& +\frac{1}{\nu}\left\|\mathcal{M}_{K}\left(\boldsymbol{f}_{h}-\boldsymbol{f}\right)\right\|_{0, K}
\end{aligned}
$$

The first term is easily bounded using the approximation properties of $\boldsymbol{f}_{h}$ and the definition of $\tau_{K}$. Next, since $\mathcal{M}_{K}\left(\boldsymbol{f}_{h}\right)=b_{K}^{p} \boldsymbol{f}_{h}$ in each $K \in \mathcal{T}_{h}$, the second term is 
bounded in the following way:

$$
\begin{aligned}
\left\|\tau_{K} \boldsymbol{f}_{h}-\frac{1}{\nu} \mathcal{M}_{K}\left(\boldsymbol{f}_{h}\right)\right\|_{0, K} & \leq\left|\tau_{K}\right|\left\|\boldsymbol{f}_{h}\right\|_{0, K}+\frac{\left\|b_{K}^{p}\right\|_{0, K}}{\nu}\left\|\boldsymbol{f}_{h}\right\|_{\mathbb{R}^{2}} \\
& \leq\left|\tau_{K}\right|\left\|\boldsymbol{f}_{h}\right\|_{0, K}+\frac{C_{K}\left|b_{K}^{p}\right|_{1, K}}{\nu|K|^{\frac{1}{2}}}\left\|\boldsymbol{f}_{h}\right\|_{0, K},
\end{aligned}
$$

where $C_{K}>0$ is the constant such that $\|v\|_{0, K} \leq C_{K}|v|_{1, K}$ for all $v \in H_{0}^{1}(K)$. Furthermore, looking carefully at the behavior of the Poincaré constant $C_{K}$ we can see (cf. [30, Thm. 1.2.5]) that $C_{K} \leq h_{K}$. On the other hand, from the definition of $b_{K}^{p}$ we have $\left|b_{K}^{p}\right|_{1, K}^{2}=\left(b_{K}^{p}, 1\right)_{K}$, and then, applying (48) we arrive at

$$
\frac{C_{K}\left|b_{K}^{p}\right|_{1, K}}{\nu|K|^{\frac{1}{2}}}\left\|\boldsymbol{f}_{h}\right\|_{0, K} \leq \frac{h_{K} \sqrt{\left(b_{K}^{p}, 1\right)_{K}}}{\nu|K|^{\frac{1}{2}}}\left\|\boldsymbol{f}_{h}\right\|_{0, K} \leq C \frac{h_{K}^{2}}{\nu}\left\|\boldsymbol{f}_{h}\right\|_{0, K} .
$$

To bound the third term in (71) we remark that function $\boldsymbol{e}:=\mathcal{M}_{K}\left(\boldsymbol{f}-\boldsymbol{f}_{h}\right)$ satisfies $-\Delta \boldsymbol{e}=\boldsymbol{f}-\boldsymbol{f}_{h}$ in $K, \boldsymbol{e}=\mathbf{0}$ on $\partial K$, and hence

$$
\|\boldsymbol{e}\|_{0, K} \leq C_{K}^{2}\left\|\boldsymbol{f}-\boldsymbol{f}_{h}\right\|_{0, K} \leq h_{K}^{2}\left\|\boldsymbol{f}-\boldsymbol{f}_{h}\right\|_{0, K} \leq C h_{K}^{3}|\boldsymbol{f}|_{1, K} .
$$

Hence, applying (70)-(73) (and assuming $h \leq 1$ ), we arrive at

$$
\begin{aligned}
\left\|\boldsymbol{u}_{1}-\boldsymbol{u}_{h}\right\|_{h}+\left\|p_{1}-p_{h}\right\|_{h} \leq & C \sup _{\left(\boldsymbol{v}_{1}, q_{1}\right) \in \mathbf{V}_{h} \times Q_{h}^{1}-\{\theta\}} \frac{\sum_{K \in \mathcal{T}_{h}} \frac{h_{K}^{2}}{\nu}\|\boldsymbol{f}\|_{1, K}\left|q_{1}\right|_{1, K}}{\left\|\boldsymbol{v}_{1}\right\|_{h}+\left\|q_{1}\right\|_{h}} \\
& \leq C \frac{h}{\sqrt{\nu}}\|\boldsymbol{f}\|_{1, \Omega},
\end{aligned}
$$

and hence (67) follows by triangular inequality and Theorem 4.8. Estimates (68) and (69) are proved as in Theorems 4.10 and 4.11 and by using (67).

\section{REFERENCES}

[1] R. Araya and F. Valentin, A multiscale a-posteriori error estimator, Comput. Methods Appl. Mech. Engrg., 194 (2005), pp. 2077-2094.

[2] C. Baiocchi, F. Brezzi, and L. P. Franca, Virtual bubbles and Galerkin-least-squares type methods (Ga. L. S.), Comput. Methods Appl. Mech. Engrg., 105 (1993), pp. 125-141.

[3] G. Barrenechea, M. Fernández, and C. Vidal, A Stabilized Finite Element Method for the Oseen Equation with Dominating Reaction, Preprint 2004-08, Departamento de Ingeniería Matemática, Universidad de Concepción, Concepción, Chile, 2004.

[4] G. BARRENECheA AND F. VALENTin, An unusual stabilized finite element method for a generalized Stokes problem, Numer. Math., 92 (2002), pp. 653-677.

[5] T. Barth, P. Bochev, M. Gunzburger, and J. Shahid, A taxonomy of consistently stabilized finite element methods for the Stokes problem, SIAM J. Sci. Comput., 25 (2004), pp. 15851607.

[6] F. BREzzI, Recent results in the treatment of subgrid scales, in CANUM 2000: Actes du 32e Congrès National d'Analyse Numérique, ESAIM Proc. II, Soc. Math. Indust., Paris, 2000, pp. 61-84.

[7] F. Brezzi, L. P. Franca, T. J. R. Hughes, and A. Russo, $b=\int g$, Comput. Methods Appl. Mech. Engrg., 145 (1997), pp. 329-339.

[8] F. Brezzi, L. Franca, And A. Russo, Further considerations on residual-free bubbles for advective-diffusive equations, Comput. Methods Appl. Mech. Engrg., 166 (1998), pp. 2533.

[9] F. Brezzi, T. J. R. Hughes, L. D. Marini, A. Russo, and E. Süli, A priori error analysis of residual-free bubbles for advection-diffusion problems, SIAM J. Numer. Anal., 36 (1999), pp. 1933-1948. 
[10] F. BrezzI, D. Marini, AND E. SüLI, Residual-free bubbles for advection-diffusion problems: The general error analysis, Numer. Math., 85 (2000), pp. 31-47.

[11] F. Brezzi and J. Pitkaranta, On the stabilization of finite element approximations of the Stokes problem, in Efficient Solutions of Elliptic Systems, W. Hackbush, ed., Notes Numer. Fluid Mech. 10, Vieweg, Braunschweig, 1984, pp. 11-19.

[12] F. Brezzi And A. Russo, Choosing bubbles for advection-diffusion problems, Math. Models Methods Appl. Sci., 4 (1994), pp. 571-587.

[13] E. Burman, M. Fernández, and P. Hansbo, Edge Stabilization for the Incompressible Navier-Stokes Equations: A Continuous Interior Penalty Finite Element Method, Tech. Report RR-5349, INRIA, Le Chesnay, France, 2004.

[14] E. Burman and P. Hansbo, A Unified Stabilized Method for Stokes' and Darcy's Equations, Tech. Report 2002-15, Chalmers Finite Element Center, Göteborg, Sweden, 2002.

[15] A. Cangiani and E. Süli, Enhanced RFB Method, Tech. Report NA-03/17, Oxford University Computing Laboratory, Oxford, UK, 2003.

[16] J. Douglas And T. Dupont, Interior penalty procedures for elliptic and parabolic Galerkin methods, in Computing Methods in Applied Sciences, R. Glowinski and J.-L. Lions, eds., Springer, Berlin, 1976, pp. 207-216.

[17] A. ERn And J.-L. Guermond, Theory and Practice of Finite Elements, Springer-Verlag, New York, 2004.

[18] C. Farhat, I. Harari, and L. Franca, The discontinuous enrichment method, Comput. Methods Appl. Mech. Engrg., 190 (2001), pp. 6455-6479.

[19] L. Franca, T. J. R. Hughes, and R. Stenberg, Stabilized finite element methods, in Incompressible Computational Fluid Dynamics, M. Gunzburger and R. Nicolaides, eds., Cambridge University Press, Cambridge, UK, 1993, pp. 87-107.

[20] L. Franca, A. Madureira, L. Tobiska, and F. Valentin, Convergence analysis of a multiscale finite element method for singularly perturbed problems, Multiscale Model. Simul., 4 (2005), pp. 839-866.

[21] L. Franca, A. Madureira, and F. Valentin, Towards multiscale functions: Enriching finite element spaces with local but not bubble-like functions, Comput. Methods Appl. Mech. Engrg., 194 (2005), pp. 3006-3021.

[22] L. P. Franca And A. Russo, Approximation of the Stokes problem by residual-free macro bubbles, East-West J. Numer. Math., 4 (1996), pp. 265-278.

[23] L. P. Franca And R. Stenberg, Error analysis of some Galerkin least squares methods for the elasticity equations, SIAM J. Numer. Anal., 28 (1991), pp. 1680-1697.

[24] V. Girault and P. A. Raviart, Finite Element Methods for Navier-Stokes Equations, Springer-Verlag, Berlin, 1986.

[25] T. Hou AND X.-H. Wu, A multiscale finite element method for elliptic problems in composite materials and porous media, J. Comput. Phys., 134 (1997), pp. 169-189.

[26] T. Hou, X.-H. Wu, AND Z. CAI, Convergence of a multiscale finite element method for elliptic problems with rapidly varying coefficients, Math. Comp., 68 (1999), pp. 913-943.

[27] T. J. R. Hughes And L. P. Franca, A new finite element formulation for computational fluid dynamics: VII. The Stokes problem with various well-posed boundary conditions: Symmetric formulations that converge for all velocity/pressure spaces, Comput. Methods Appl. Mech. Engrg., 65 (1987), pp. 85-96.

[28] T. J. R. Hughes, L. P. Franca, and M. Balestra, A new finite element formulation for computational fluids dynamics: V. Circumventing the Babuska-Brezzi condition: A stable Petrov-Galerkin formulation of the Stokes problem accommodating equal-order interpolations, Comput. Methods Appl. Mech. Engrg., 62 (1987), pp. 85-99.

[29] N. Kechar And D. Silvester, Analysis of a locally stabilized mixed finite element method for the Stokes problem, Math. Comp., 58 (1992), pp. 1-10.

[30] P. A. Raviart And J.-M. Thomas, Introduction à l'Analyse Numérique des Équations aux Dérivées Partielles, Masson, Paris, 1983.

[31] A. Russo, Bubble stabilization of finite element methods for the linearized incompressible Navier-Stokes equations, Comput. Methods Appl. Mech. Engrg., 132 (1996), pp. 335-343.

[32] G. SANGALLI, Global and local error analysis for the residual-free bubbles method applied to advection-dominated problems, SIAM J. Numer. Anal., 38 (2000), pp. 1496-1522.

[33] V. Tноме́e, Galerkin Finite Element Methods for Parabolic Problems, Springer-Verlag, Berlin, 1997.

[34] L. Tobiska And R. Verfürth, Analysis of a streamline diffusion finite element method for the Stokes and Navier-Stokes equations, SIAM J. Numer. Anal., 33 (1996), pp. 107-127. 